\title{
SUPPORTING INFORMATION \\ Exciton Delocalization in Indolenine Squaraine Aggregates Templated by DNA Holliday Junction Scaffolds
}

${ }^{1}$ Olga A. Mass; ${ }^{1}$ Christopher K. Wilson; ${ }^{1}$ Simon K. Roy; ${ }^{1}$ Matthew S. Barclay; ${ }^{1}$ Lance K. Patten;

${ }^{2}$ Ewald A.Terpetschnig; *1,3 Jeunghoon Lee; *1 Ryan D. Pensack; *1,4Bernard Yurke;

*1,4William B. Knowlton.

${ }^{1}$ Micron School of Materials Science \& Engineering, ${ }^{3}$ Department of Chemistry and Biochemistry, ${ }^{4}$ Department of Electrical \& Computer Engineering, Boise State University, Boise, Idaho 83725, United States

${ }^{2}$ SETA BioMedicals, LLC, 2014 Silver Court East, Urbana, IL 61801, United States

Corresponding authors:

*jeunghoonlee@boisestate.edu

*ryanpensack@boisestate.edu

*bernardyurke@boisestate.edu

*bknowlton@boisestate.edu

\section{Table of Contents}

SI1 Covalent Attachment of Square-660 to DNA $\quad$ S2

SI2 Qualitative Nondenaturing Polyacrylamide Gel Electrophoresis S3

SI3 Melting Profiles $\quad$ S4

SI4 Fluorescence and Fluorescence Suppression S9

$\begin{array}{ll}\text { SI5 Fluorescence Quantum Yield } & \text { S10 }\end{array}$

SI6 Spectral Properties of Free Square-660 Dye and DNA-Dye Monomer Constructs $\quad \mathrm{S} 12$

$\begin{array}{ll}\text { SI7 Fitting Absorbance Data } & \text { S12 }\end{array}$

SI8 Circular Dichroism Full Spectra $\quad$ S15

SI9 Fitting Absorption and CD using KRM Model $\quad$ S16

SI10 Influence of DNA HJ Conformations on Squaraine Aggregation $\quad$ S29

$\begin{array}{lll}\text { SI11 References } & \text { S34 }\end{array}$ 
Supporting Information 1: Covalent Attachment of Square-660 to DNA

Square-660 dyes were attached to DNA by means of amidation reaction between Square-660NHS ester and the amino group of a non-nucleosidic serinol spacer (Fig. S1). The attachment chemistry and the subsequent dual HPLC purification were carried out at Bio-Synthesis.

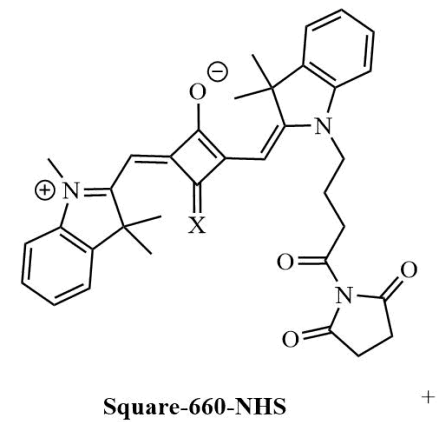

Square-660-NHS

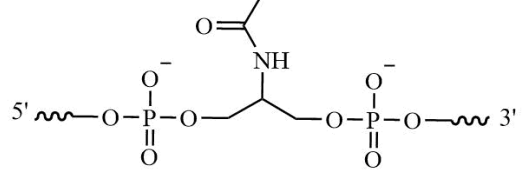

amino serinol spacer within oligonucleotide

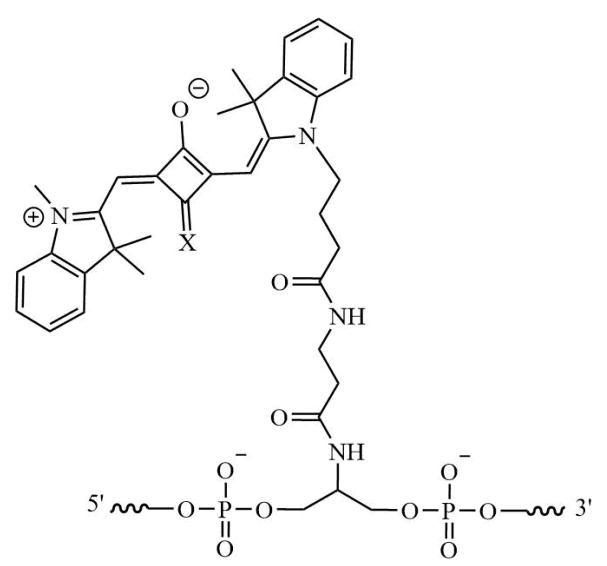

Figure S1. Post-modification of oligonucleotide with Square-660-NHS $\left[\mathrm{X}=\mathrm{O}, \mathrm{NR}^{1}, \mathrm{C}(\mathrm{CN})_{2}\right]$, with structural details provided by SETA BioMedicals and Bio-Synthesis shown. 
Supporting Information 2: Qualitative Nondenaturing Polyacrylamide Gel Electrophoresis

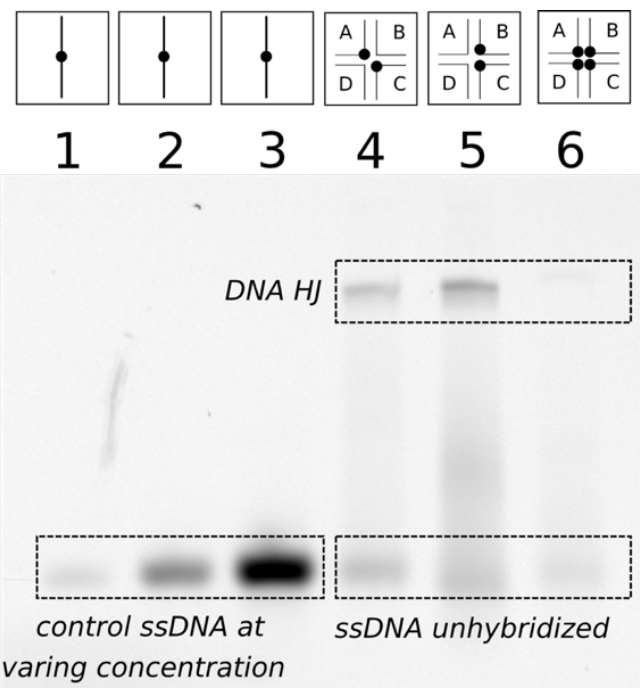

bromophenol blue
Table S1.

\begin{tabular}{lcc}
\hline lane & sample & ${ }^{a}[\mathbf{C}], \boldsymbol{\mu M}$ \\
\hline 1 & ${ }^{b}$ SSDNA-SQ & 0.0075 \\
2 & ${ }^{b}$ SSDNA-SQ & 0.075 \\
3 & ${ }^{b}$ SSDNA-SQ & 0.15 \\
4 & SQ-AC & 0.15 \\
5 & SQ-BC & 0.15 \\
6 & SQ-ABCD & 0.15
\end{tabular}

${ }^{a}$ Sample concentration in $10 \mu \mathrm{L}$ loading volume. ${ }^{b}$ Strand B functionalized with Square-660.

Figure S2. Fluorescent image of $15 \%$ nondenaturing PAGE $1.5 \mathrm{~mm}$ run at $150 \mathrm{~V}, 17{ }^{\circ} \mathrm{C}$ in $1 \times$ TBE, $15 \mathrm{mM} \mathrm{MgCl}_{2}$. Note that as fluorescence of squaraine aggregates is highly suppressed, the visibility of the corresponding DNA HJ gel bands is poor.

\section{Polyacrylamide gel electrophoresis and quantification}

Nondenaturing PAGE was carried out to estimate the amounts of ssDNA remained in each squaraine aggregate sample after hybridization. The $15 \%$ nondenaturing electrophoresis gel, 1.5 mm was casted in $1 \times$ TBE $15 \mathrm{mM} \mathrm{MgCl} 2$. DNA samples were diluted 10-fold in Loading Buffer (Ficoll (SigmaAldrich) 20\% v/v, bromophenol blue (SigmaAldrich) 20\% v/v). The DNA HJ samples were loaded on the gel at DNA construct concentration $0.15 \mu \mathrm{M}$ alongside with three ssDNA controls at $0.0075,0.075$ and $0.15 \mu \mathrm{M}$. PAGE was run for $1 \mathrm{~h} 40$ min at $150 \mathrm{~V}$ constant voltage at $17{ }^{\circ} \mathrm{C}$ constant temperature in running buffer $1 \times \mathrm{TBE}, 15 \mathrm{mM} \mathrm{MgCl}_{2}$. The electrophoresed gel was scanned in FluorChem Q imager (Alpha Innotech) in the Cy5-channel 
(ex. $632 \mathrm{~nm}$; em. $691 \mathrm{~nm}$ ). The amounts of ssDNA remained in the aggregate samples SQ-AC, SQ-BC and SQ-ABCD were quantified against ssDNA, $0.0075 \mu \mathrm{M}$ (lane 1) using ImageJ 1.53a.

\section{Supporting Information 3: Melting Profiles}

Melting profiles of squaraine-DNA constructs were recorded using a Varian Cary5000 spectrophotometer equipped with a thermal probe (Agilent Technologies Cary Temperature Controller G9808). Samples were degassed for $5 \mathrm{~min}$ at room temperature. Samples were equilibrated at $25{ }^{\circ} \mathrm{C}$ for 2 min before starting a temperature ramp of $1{ }^{\circ} \mathrm{C} / \mathrm{min}$ over a temperature range from $25{ }^{\circ} \mathrm{C}$ to $95{ }^{\circ} \mathrm{C}$. Absorption was monitored at $260 \mathrm{~nm}$. The thermal denaturation temperatures $\left(\mathrm{T}_{\mathrm{m}}\right)$ of squaraine-DNA constructs were determined by fitting a Gaussian to the first derivative of absorption in OriginPro 2019.

For comparison purposes, the thermal profiles in the presence of $\mathrm{MgCl}_{2}$ were recorded for unmodified DNA HJ and squaraine aggregates SQ-A, SQ-AC, and SQ-ABCD (Fig. S3) and the thermal profiles in the presence of $\mathrm{NaCl}$ were recorded for unmodified DNA HJ and squaraine aggregates SQ-A, SQ-AC, SQ-BC, SQ-BD, and SQ-ABCD (Fig. S4). The melting temperatures are summarized in Table $\mathbf{S 2}$.

The squaraine constructs in the presence of $\mathrm{NaCl}$ melted $6-13{ }^{\circ} \mathrm{C}$ lower than corresponding squaraine constructs in $\mathrm{MgCl}_{2}$. According to the melting profiles, unmodified DNA HJ and squaraine constructs in $\mathrm{NaCl}$ exhibited noncooperative unfolding characterized with more than one transition partially resolved with Gauss curve fitting. This melting behavior is consistent with that previously observed for DNA HJ in the open conformation. ${ }^{1-2}$ Because the open conformation of DNA HJ lacks coaxial base pair stacking, its DNA HJ arms unfold more independently through a series of transitions ${ }^{2}$ and at the lower temperatures relative to the 
stacked conformation. Melting experiments in the presence of $\mathrm{NaCl}$ experimentally support that in $\mathrm{MgCl}_{2}$ squaraine constructs are templated by the stacked conformation of DNA HJ. 

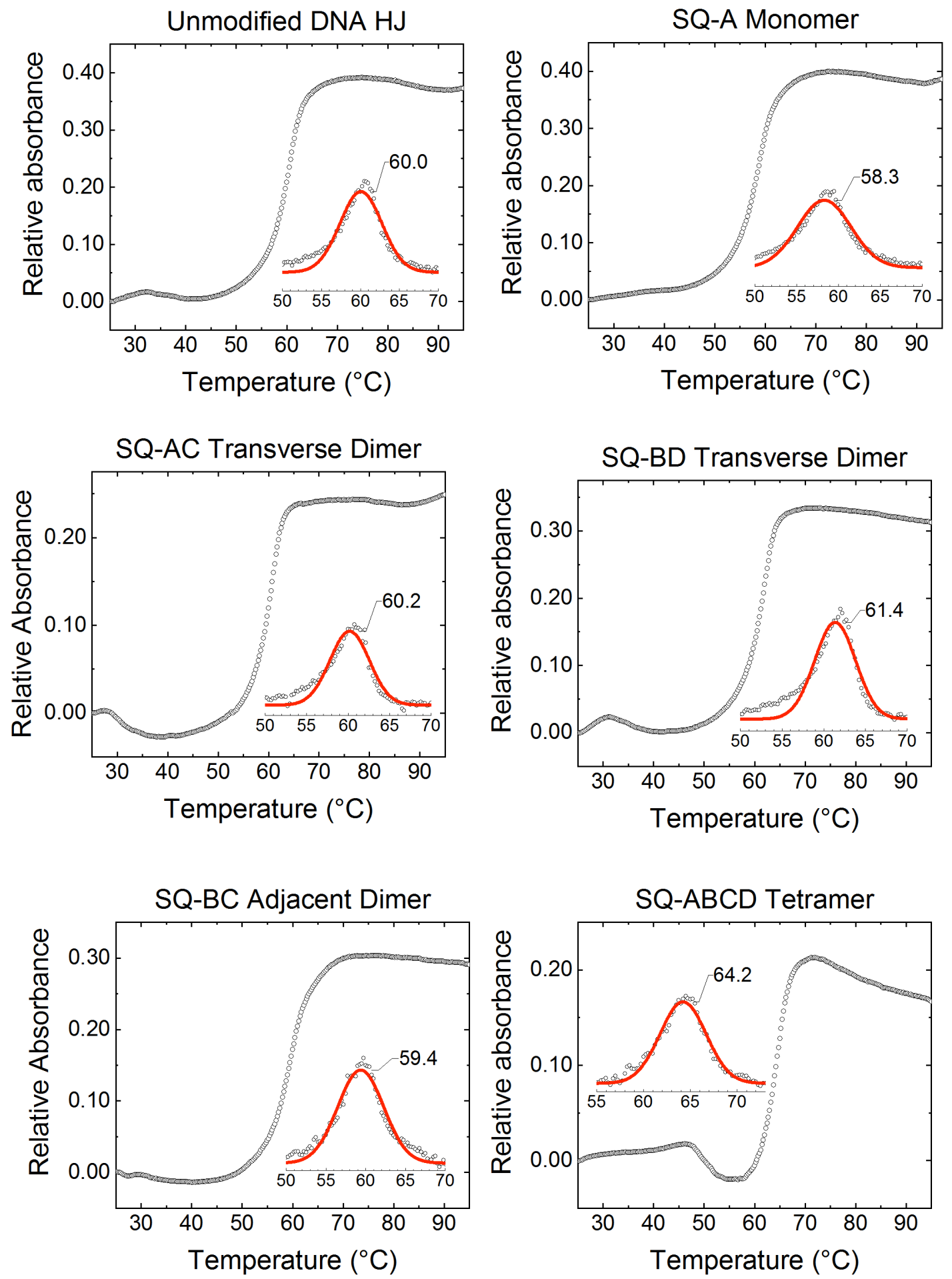

Figure S3. Melting profiles of unmodified DNA HJ and squaraine aggregates in the presence of $\mathrm{MgCl}_{2}$. The thermal denaturation was monitored in $1 \times \mathrm{TBE}, 15 \mathrm{mM} \mathrm{MgCl}_{2}$ containing $1.5 \mu \mathrm{M}$ HJ DNA construct. The inserts show first derivative as a function of temperature and a fitted Gaussian curve. 

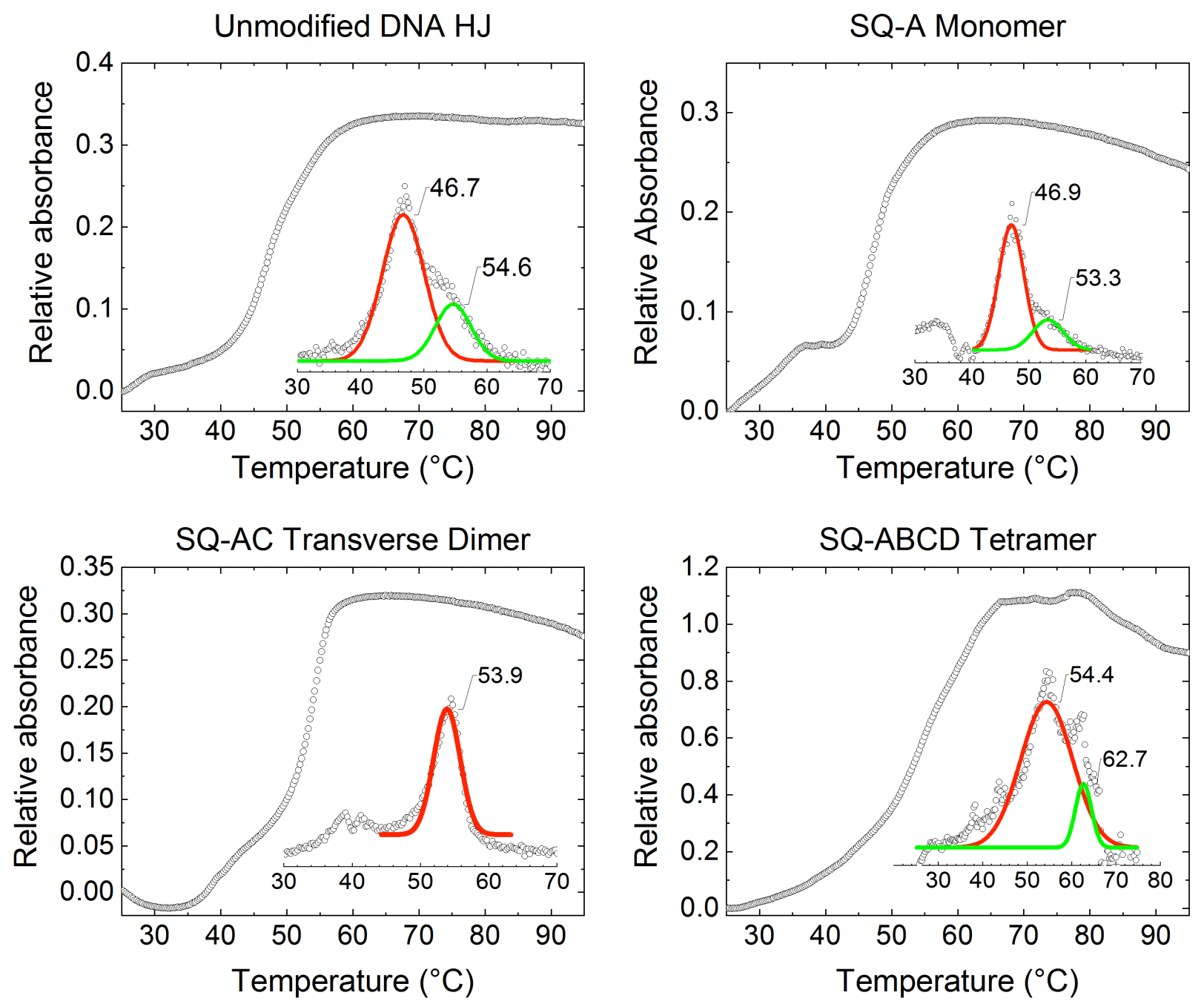

Figure S4. Melting profiles of unmodified DNA HJ and squaraine aggregates in the presence of $\mathrm{NaCl}$. The thermal denaturation was monitored in $1 \times \mathrm{TBE}, 100 \mathrm{mM} \mathrm{NaCl}$ containing $1.5 \mu \mathrm{M} \mathrm{HJ}$ DNA construct. The inserts show first derivative as a function of temperature and fitted Gaussian curve(s). 
Table S2. Melting temperatures of squaraine aggregates.

\begin{tabular}{lc} 
Construct & $\mathrm{T}_{\mathrm{m},}{ }^{\circ} \mathrm{C}$ \\
\hline \hline Unmodified DNA HJ, $15 \mathrm{mM} \mathrm{MgCl}_{2}$ & 60.0 \\
Monomer SQ-A, $15 \mathrm{mM} \mathrm{MgCl} 2$ & 58.3 \\
Transverse dimer SQ-AC, $15 \mathrm{mM} \mathrm{MgCl}_{2}$ & 60.2 \\
Transverse dimer SQ-BD, $15 \mathrm{mM} \mathrm{MgCl}_{2}$ & 61.4 \\
Adjacent dimer SQ-BC, $15 \mathrm{mM} \mathrm{MgCl} 2$ & 59.4 \\
Tetramer SQ-ABCD, $15 \mathrm{mM} \mathrm{MgCl} 2$ & 64.2 \\
\hline Unmodified DNA HJ, $100 \mathrm{mM} \mathrm{NaCl}$ & 46.7 \\
Monomer SQ-A, $100 \mathrm{mM} \mathrm{NaCl}$ & 46.9 \\
Transverse dimer SQ-AC, $100 \mathrm{mM} \mathrm{NaCl}$ & 53.9 \\
Tetramer SQ-ABCD,100 mM NaCl & 54.4
\end{tabular}


Supporting Information 4: Fluorescence and Fluorescence Suppression

Fluorescence spectra were collected and scaled by the absorptance at the excitation maximum. The absorptance is defined as

$$
A=1-T=1-10^{-a b s}
$$

where $\mathrm{A}$ is absorptance at the excitation wavelength $\left(\lambda_{\text {exc }}\right), \mathrm{T}-$ transmitted light, and abs absorption at $\lambda_{\text {exc. }}$

The area under emission curve between $665-740 \mathrm{~nm}$ of each aggregate was integrated in OriginPro2019. Obtained areas of integration were used to calculate fluorescence suppression according to the following formula:

$$
\% F L \text { Suppression }=\frac{\int F L_{\text {monomer }}-\int F L_{\text {aggregate }}}{\int F L_{\text {monomer }}} \times 100 \%
$$

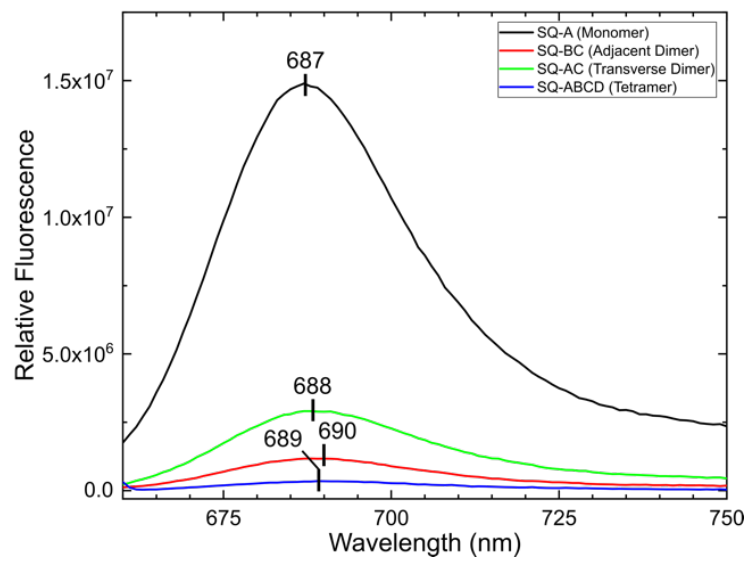

Figure S5. Scaled fluorescence emission of squaraine monomer, adjacent dimer, transverse dimer, and a tetramer recorded in $1 \times \mathrm{TBE}, 15 \mathrm{mM} \mathrm{MgCl}_{2}$ containing $0.5 \mu \mathrm{M}$ HJ DNA construct at room temperature. Samples were excited at $\lambda_{\mathrm{exc}}=650 \mathrm{~nm}$. The fluorescence was normalized by the absorptance at the excitation wavelength. 
Table S3. Fluorescence suppression of squaraine aggregates.

\begin{tabular}{lcc}
\hline \multicolumn{1}{c}{ Construct } & Integrated area & FL suppression, \% \\
\hline SQ-A (monomer) & $117 \times 10^{6}$ & $\mathrm{n} / \mathrm{a}$ \\
SQ-AC (transverse dimer) & $22.9 \times 10^{6}$ & 80 \\
SQ-BC (adjacent dimer) & $9.25 \times 10^{6}$ & 92 \\
SQ-ABCD (tetramer) & $2.87 \times 10^{6}$ & 98
\end{tabular}

\section{Supporting Information 5: Fluorescence Quantum Yield}

Fluorescence quantum yield (FQY) values were obtained by measuring the fluorescence emission spectrum of the unknown species relative to standard reference material of known quantum yield $\left(\Phi_{\text {ref. }}\right)$. The unknown FQY $\left(\Phi_{\text {unk. }}\right)$ is determined according to the following equation,

$$
\Phi_{\text {unk. }}=\Phi_{\text {ref. }}\left(\frac{I_{\text {unk. }}}{I_{\text {ref. }}}\right)\left(\frac{A_{\text {ref. }}^{\lambda}}{A_{\text {unk. }}^{\lambda}}\right)\left(\frac{n_{\text {unk. }}{ }^{2}}{n_{\text {ref. }}{ }^{2}}\right)
$$

where $I_{\text {unk. }}$ and $I_{\text {ref. }}$ are the integrated fluorescence emission intensities of the unknown and reference samples, respectively, the $A^{\lambda}$ term denotes the absorbance (in units of OD) at the fluorescence excitation wavelength $(\lambda)$, and the $n$ terms are the refractive indices of the solvents used to make the samples.

Relative FQY measurements for the monomer SQ-A in $1 \times$ TBE buffer with $15 \mathrm{mM} \mathrm{MgCl}_{2}$ were performed on a on a Horiba Fluorolog 3 spectrofluorometer (Horiba Scientific) using a $1 \mathrm{~cm}$ path length quartz cell (Starna). Typical scan parameters were $1 \mathrm{~nm}$ steps and $0.5 \mathrm{~s}$ integration time. The optical density was kept at or below 0.05 at the excitation wavelength. A sample of doublestranded DNA-templated Cy5 dye monomer in $1 \times \mathrm{TAE}$ buffer with $0 \mathrm{mM} \mathrm{MgCl} 2$ was used as a relative FQY standard $\left(\Phi_{\text {ref. }}=0.29^{3}\right)$. The excitation wavelength was set to $615 \mathrm{~nm}$ and remained unchanged between measurements on the Cy5 monomer and Square-660 monomer 
solutions to ensure an equivalent intensity of light was incident on the sample for each measurement. The slit widths for the measurements were chosen to maximize the signal from the monomer while staying within the linear range of the detection system. Both the excitation and emission monochromator slit widths were set to a $5.00 \mathrm{~nm}$ bandpass. The aqueous TAE and TBE buffer solvents have nearly identical refractive indices, therefore, the right-most term was set equal to 1 .

The FQY values of Square-660 aggregates were obtained separately, using the SQ-A monomer sample as the reference as described above with the excitation wavelength of $\lambda=650$ nm. All fluorescence spectra were intensity corrected.

Table S4. Fluorescence Quantum Yield of Square-660 aggregates.

\begin{tabular}{l|l} 
Construct & $\Phi_{\mathrm{F}}{ }^{a}$ \\
\hline SQ-A (monomer) & 0.37 \\
SQ-AC (transverse dimer) & 0.038 \\
SQ-BC (adjacent dimer) & 0.023 \\
SQ-ABCD (tetramer) & 0.006
\end{tabular}

${ }^{a}$ Fluorescence quantum yield measured in $1 \times \mathrm{TBE}, 15 \mathrm{mM} \mathrm{MgCl}_{2}$. 
Supporting Information 6: Spectral Properties of Free Square-660 Dye and DNA-Dye Monomer Constructs

a

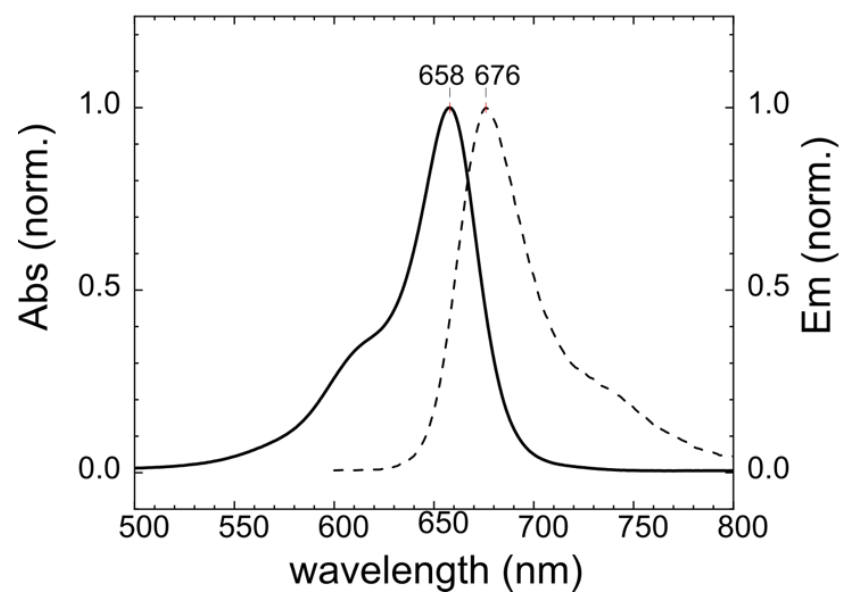

b

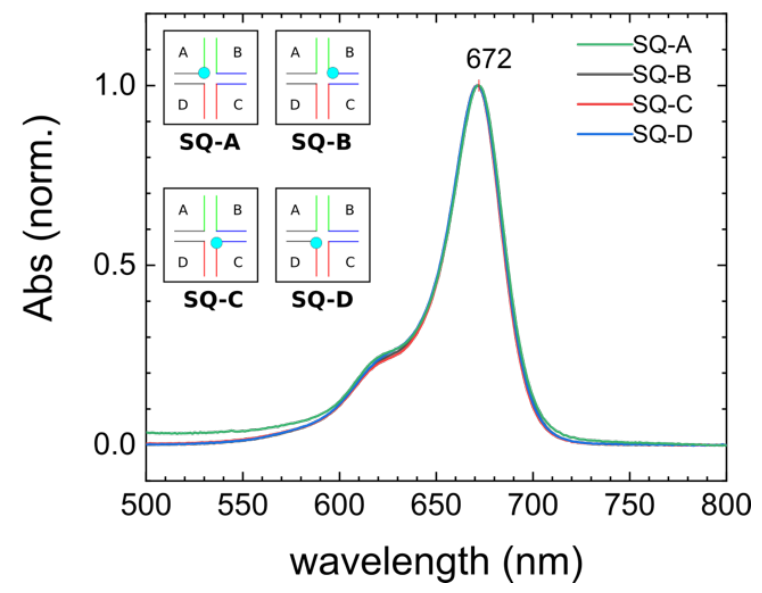

Figure. S6. (a) Absorption (solid line) and emission (dash line) of free dye Square-660 in PBS buffer, pH 7.0. (b) Absorption of squaraine monomers SQ-A, SQ-B, SQ-C and SQ-D templated by DNA HJ recorded in $1 \times \mathrm{TBE}, 15 \mathrm{mM} \mathrm{MgCl}_{2}$ at room temperature. The DNA-dye monomer construct concentration was $1.5 \mu \mathrm{M}$.

\section{Supporting Information 7: Fitting Absorbance Spectra}

Absorption spectra for each construct were analyzed by fitting each data set into a series of three Gaussian curves verses a series of four Gaussian curves using OriginPro2019 software. The 3component Gaussian fit afforded a larger residual in the $680-700 \mathrm{~nm}$ range compared to the 4component fit. This result mathematically justifies a four-component Gaussian fit with the lowest-energy absorption band $\mathrm{A}_{1}$. 

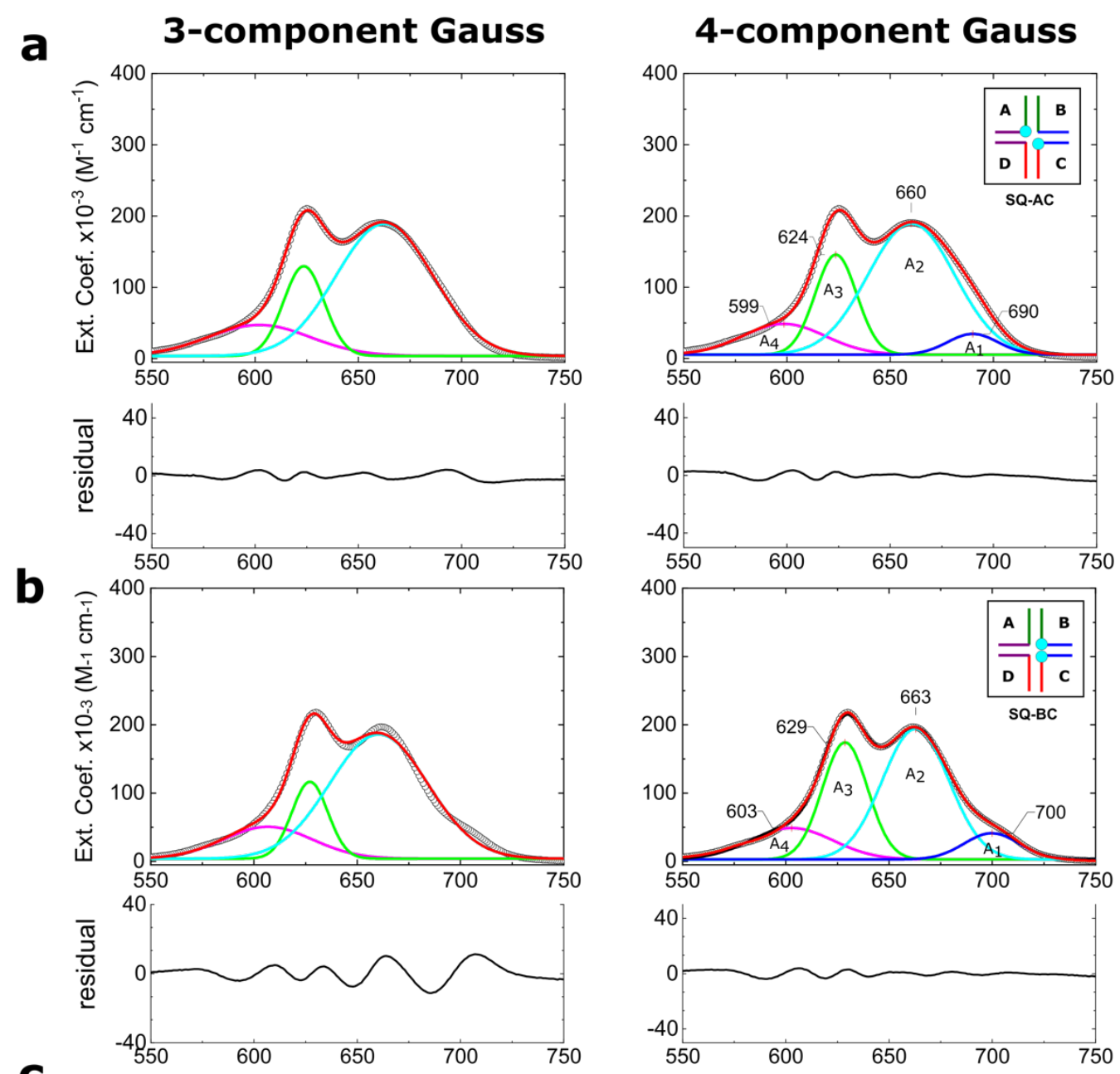

$\mathbf{C}$
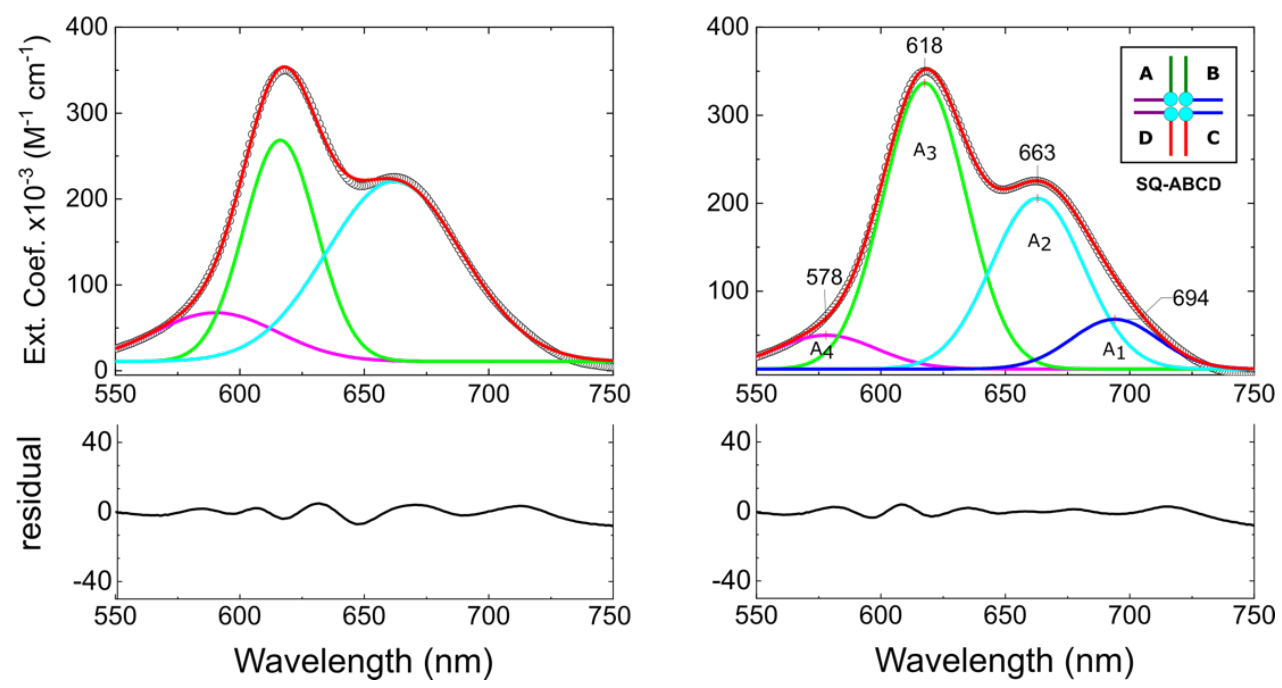

Figure S7. Gaussian 3-component (left panel) vs 4-component (right panel) fits of the a monomer SQ-A; b - transverse dimer SQ-AC; $\mathbf{c}$ - adjacent dimer SQ-BC; and $\mathbf{d}$ - tetramer SQABCD. 


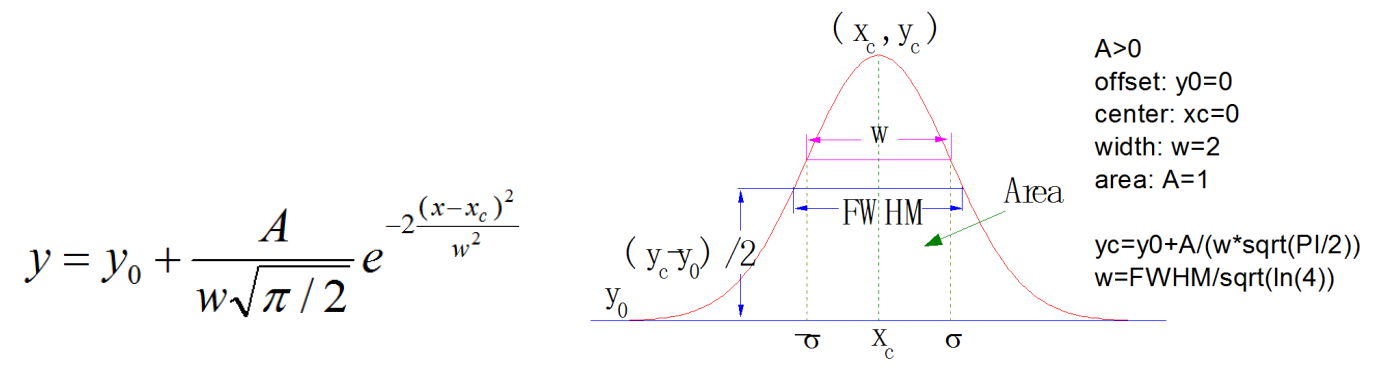

Table S5. Gaussian fitting parameters of squaraine aggregate absorption spectra.

\begin{tabular}{lc|c|r|r|r|r|} 
& & $\begin{array}{c}\mathrm{x}_{\mathrm{c}} \\
(\mathrm{nm})\end{array}$ & $\begin{array}{c}\mathrm{w} \\
(\mathrm{nm})\end{array}$ & $\begin{array}{c}\mathrm{FWHM} \\
(\mathrm{nm})\end{array}$ & $\begin{array}{c}\text { Area, } \\
\left(\mathrm{nm}^{2}\right)\end{array}$ & $\begin{array}{c}\text { Adjusted } \\
\mathrm{R}^{2}\end{array}$ \\
\hline & & & & & & \\
SQ-AC & & & & & & \\
& $\mathrm{A}_{1}$ & 690 & 24 & 29 & 900 & \\
& $\mathrm{~A}_{2}$ & 660 & 42 & 50 & 9800 & \\
& $\mathrm{~A}_{3}$ & 624 & 20 & 24 & 3600 & \\
& $\mathrm{~A}_{4}$ & 599 & 40 & 47 & 2200 & 0.999 \\
& & & & & & \\
SQ-BC & & & & & & \\
& $\mathrm{A}_{1}$ & 700 & 27 & 31 & 1280 & \\
& $\mathrm{~A}_{2}$ & 663 & 32 & 38 & 7800 & \\
& $\mathrm{~A}_{3}$ & 629 & 22 & 26 & 4700 & \\
& $\mathrm{~A}_{4}$ & 603 & 40 & 47 & 2300 & 0.999 \\
& & & & & & \\
SQ-ABCD & & & & & & \\
& $\mathrm{A}_{1}$ & 694 & 35 & 41 & 2500 & \\
& $\mathrm{~A}_{2}$ & 663 & 37 & 44 & 9000 & 1.000 \\
& $\mathrm{~A}_{3}$ & 618 & 33 & 39 & 14000 & \\
& $\mathrm{~A}_{4}$ & 578 & 40 & 47 & 1900 &
\end{tabular}


Supporting Information 8: Circular Dichroism Full Spectra
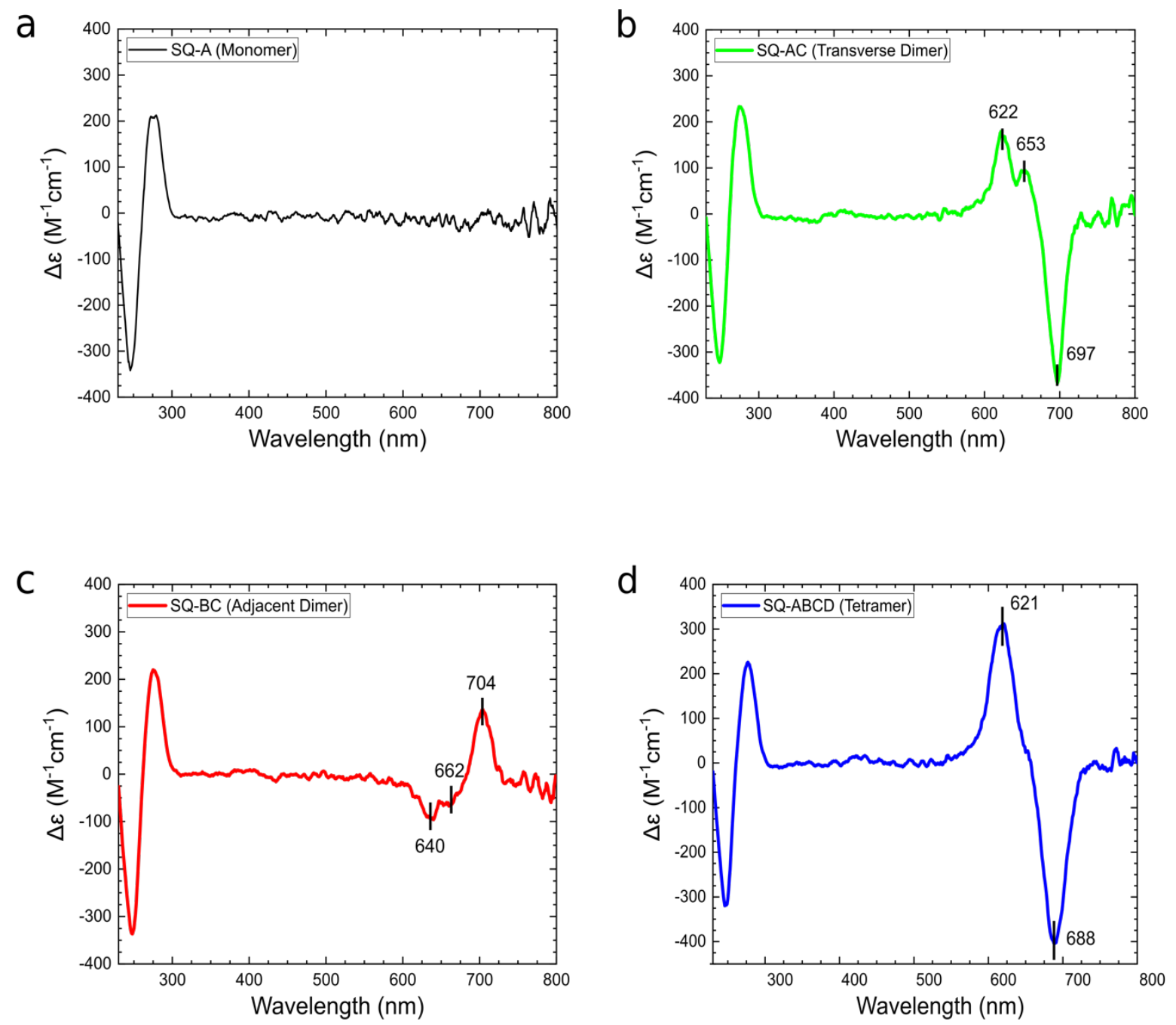

Figure S8. Full CD spectra of the DNA-Square 660 dye constructs recorded in $1 \times$ TBE, $15 \mathrm{mM}$ $\mathrm{MgCl}_{2}$ at room temperature. The DNA-dye construct concentration was $1.5 \mu \mathrm{M}$. 


\section{Supporting Information 9: Fitting Absorption and CD using KRM Model}

The behavior of molecular, or Frenkel, excitons in dye aggregate system is well approximated by an augmented Frenkel Hamiltonian that includes exciton and vibron components:

$$
H=H^{(e)}+H^{(v)}
$$

The $H^{(\mathrm{e})}$ component includes excitonic hopping parameter $J_{m, n}$ (i. e., a single exciton transition dipole-dipole coupling between dyes on sites $\mathrm{m}$ and $\mathrm{n}$ ).

Insight into the meaning of the $J_{m, n}$ and the $J_{0}$ parameter is obtained by comparing the dipoledipole approximation of $J_{m, n}$ with the extended dipole approximation. When intermolecular distances are much greater than dye size, the $J_{m, n}$ can be expressed in a point dipole-dipole interaction form ${ }^{4-6}$ representing the interaction between a pair of molecular transition dipoles $\mu_{m}$ and $\mu_{n}$ for the dyes at sites $m$ and $n$ :

$$
J_{m, n}=\frac{1}{4 \pi \varepsilon \varepsilon_{0}}\left(\frac{\mu_{m} \cdot \mu_{n}}{\left|\boldsymbol{R}_{m, n}\right|^{3}}-\frac{\left(\mu_{m} \cdot \boldsymbol{R}_{m, n}\right)\left(\mu_{n} \cdot \boldsymbol{R}_{m, n}\right)}{\left|\boldsymbol{R}_{m, n}\right|^{5}}\right)
$$

where $\boldsymbol{R}_{m, n}$ - the position vector between the dye centers dyes $m$ and $n$. (further $\boldsymbol{R}_{1,2}$ between two dyes)

In the extended dipole approximation, ${ }^{7}$ the Coulomb energy between a pair of dipoles is given in Standard International units by

$$
J_{m, n}=\frac{\delta^{2}}{4 \pi \epsilon_{0} \epsilon_{r}}\left(\frac{1}{|\boldsymbol{r}-\boldsymbol{r}|}-\frac{1}{|\boldsymbol{r}-\boldsymbol{s}|}-\frac{1}{|s-\boldsymbol{r}|}+\frac{1}{|s-s|}\right)
$$

where $\delta$ is the oscillating point charge in Coulombs, $\epsilon_{0}$ is the permittivity of the vacuum $\left(\epsilon_{0}=\right.$ $\left.8.85 \times 10^{-12} \mathrm{~m}^{-3} \mathrm{~kg}^{-1} \mathrm{~s}^{4} \mathrm{~A}^{2}\right), \epsilon_{r}$ is the relative dielectric constant of the medium, $\boldsymbol{r}$ and $\boldsymbol{s}$ are the location of the "+" and "- "charges on molecule 1 and similarly for molecule 2 . The distance units are meters and the exchange energy $J_{m, n}$ unit are Joules. 
The transition dipole moment is given by $\mu=\delta l$ where $l$ is the distance in meters between the two point charges on a given molecule, and $r$ is given by $\epsilon_{r}=n^{2}$ where $n$ is the index of refraction of the medium.

Then the equation (S3) can be rewritten as:

$J_{12}=\frac{J_{0}}{l^{2}}\left(\frac{1}{|r-r|}-\frac{1}{|r-s|}-\frac{1}{|s-r|}+\frac{1}{|s-s|}\right)$

where $J_{0}=\frac{\mu^{2}}{4 \pi \epsilon_{0} n^{2}}$

The quantity $J_{0}$ has units of $\mathrm{J} \cdot \mathrm{m}^{3}$.

When the position vectors $\boldsymbol{r}$ and $\boldsymbol{s}$ are rewritten as:
$\boldsymbol{r}=\boldsymbol{R}+\frac{l}{2} n$
$s=R-\frac{l}{2} n$
$\boldsymbol{r}=\boldsymbol{R}+\frac{l}{2} \boldsymbol{n}$
$s=R-\frac{l}{2} n$

And when $|\boldsymbol{R}-\boldsymbol{R}| \gg l$, equation (eq S4) reduces to:

$J_{12}=\frac{J_{0}}{|\boldsymbol{R}-\boldsymbol{R}|^{3}}[\boldsymbol{n} \cdot \boldsymbol{n}-3(\boldsymbol{n} \cdot \boldsymbol{n})(\boldsymbol{n} \cdot \boldsymbol{n})] \quad($ eq S6)

where $n=\frac{R-R}{|R-R|}$

Thus, $J_{0}$ is the same constant in both the point dipole-dipole approximation and in the extended dipole approximation. 
The theoretical absorbance as a function of energy, for comparison with experimental data, was computed from the line spectra obtained by diagonalizing the system Holstein-like Hamiltonian $^{8}$ by convolution with a Gaussian as:

$$
A(E)=\sum_{i} \frac{\gamma_{i}}{\sqrt{2 \pi \Gamma^{2}}} \exp \left(-\frac{\left(E-E_{i}\right)^{2}}{2 \Gamma^{2}}\right)
$$

Where the $\gamma_{i}$ is the transition rates between the ground state $E$ and the state having the eigenenergy $E_{i}$, and $\Gamma$ is a linewidth.

Similarly, the CD absorbance as a function of energy was computed as:

$$
A_{C D}(E)=\sum_{i} \frac{\gamma_{i}^{C D}}{\sqrt{2 \pi \Gamma^{2}}} \exp \left(-\frac{\left(E-E_{i}\right)^{2}}{2 \Gamma^{2}}\right)
$$

The phenomenological constants used in the theoretical model are shown in Tbl. S6. To fit the monomer and then dimers and a tetramer data, we considered the length of the squaraine transition dipole moment to be $1.3 \mathrm{~nm}$ (which is slightly shorter than the length of an indolenine squaraine dye), the linewidth $\Gamma$ to be $0.033 \mathrm{eV}$, and number of vibronic states $\mathrm{n}_{\mathrm{v}}$ to be 3 .

The transition dipole moment was determined using the following expression: ${ }^{9}$

$$
M_{01}=9.58 \times 10^{-2}\left(\frac{\left(2 n^{2}+1\right)^{2}}{9 n^{3}} \int \frac{\epsilon(v)}{v} d v\right)^{\frac{1}{2}}
$$

$M_{01}$ - transition dipole moment in Debye; $n$ - refractive index of water $\left(\left(\mathrm{n}_{\text {water }}=1.3327\right)\right.$.

Note that $\mu$ has units of Coulomb meters while $M_{01}$ has units of Debye. The conversion relation between the two is 1 Debye $=3.33564 \times 1030 \mathrm{C} \cdot \mathrm{m}$. 
Characteristic exciton hopping parameter $J_{0}$ was calculated from $M_{01}$ according to the equation (S5) to be $48 \mathrm{meV} \cdot \mathrm{nm}^{3}$ for SQ monomer and $49 \mathrm{meV} \cdot \mathrm{nm}^{3}$ for Cy5 monomer (Tbl. S7), and its value was used as a fitting parameter in the calculations of dimers and a tetramer (Tbl. S6).

As a measure the goodness of the fits, we evaluated the overlap integrals of the experimental $\begin{array}{lllll}\text { spectra with the } & \text { theoretical }\end{array}$ Letting $\int S_{a b, e x}(E), S_{a b, t h}(E), S_{c d, e x}(E)$, and $S_{c d, t h}(E)$ denote respectively the experimental absorbance spectrum, theoretical absorbance spectrum, experimental CD spectrum, and theoretical CD spectrum where $\boldsymbol{E}$ is energy, the normalized absorbance overlap integral $O I_{A B}$ of the spectrum is defined by

$$
O I_{A B}=\frac{\int S_{a b, e x}(E) S_{a b, t h}(E) d E}{\sqrt{\int S_{a b, e x}^{2}(E) d E} \sqrt{\int S_{a b, t h}^{2}(E) d E}}
$$

and the normalized overlap integral for the $\mathrm{CD}$ spectrum $O I_{C D}$ is defined by

$$
O I_{C D}=\frac{\int S_{c d, e x}(E) S_{c d, t h}(E) d E}{\sqrt{\int S_{c d, e x}^{2}(E) d E} \sqrt{\int S_{c d, t h}^{2}(E) d E}}
$$

As an overall goodness parameter we introduce

$$
O I_{t o t}=\frac{1}{2}\left(O I_{a b}+O I_{c d}\right)
$$

In addition, mean-square deviation of absorbance and $\mathrm{CD}$ were utilized:

$$
\begin{aligned}
& m s_{a b s}=\sum_{i}\left[S_{a b, e x}(E)-S_{a b, t h}(E)\right]^{2} \\
& m s_{c d}=\sum_{i}\left[S_{c d, e x}(E)-S_{c d, t h}(E)\right]^{2} \\
& \text { Fitness }=w_{1}(1-r)^{2}+w_{2}\left(1-O I_{A B}\right)^{2}+w_{3}\left(1-O I_{C D}\right)^{2}+w_{4} m s_{a b s}+w_{5} m s_{c d}
\end{aligned}
$$


where $w_{1}, w_{2}, w_{3}$, and $w_{4}$ are user selected weights. For a typical run the weights were chosen as $w_{1}=1, w_{2}=w_{3}=0$, and $w_{4}=w_{5}=1$.

The resulting outputs of the fit provide information regarding the angles and position of the dyes relative to each other. Given in spherical coordinates, the zenith $\left(\theta_{i}\right)$ and azimuth $\left(\varphi_{i}\right)$ angles are given in degrees. The Cartesian components of the orientation vector for a dye are given in terms of $\theta_{i}$ and $\varphi_{i}$ by the following set of equations:

$$
\begin{gathered}
n_{x}=\sin \left(\theta_{i}\right) \cos \left(\varphi_{i}\right) \\
n_{y}=\sin \left(\theta_{i}\right) \sin \left(\varphi_{i}\right), \\
n_{z}=\cos \left(\theta_{i}\right)
\end{gathered}
$$

The positions of the dyes are given in angstroms and listed in Tbls. S9 and S10.

A center-to-center distance $R$ between two dyes was calculated as:

$$
R=\sqrt{\left(x_{2}-x_{1}\right)^{2}+\left(y_{2}-y_{1}\right)^{2}+\left(z_{2}-z_{1}\right)^{2}}
$$

A slip angle $\theta_{s}$ was calculated as:

$$
\theta_{s}=\cos ^{-1}[n \cdot n]
$$

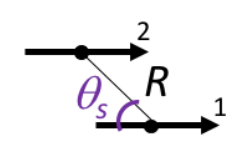

Where $\boldsymbol{n}_{\boldsymbol{1}}$ is a unit orientation vector

$$
n=\sin \left(\theta_{1}\right) \cos \left(\varphi_{1}\right) \hat{x}+\sin \left(\theta_{1}\right) \sin \left(\varphi_{1}\right) \hat{y}+\cos \left(\theta_{1}\right) \hat{z}
$$

And where $\boldsymbol{n}$ is a unit vector connecting dye centers

$$
\boldsymbol{n}=\frac{1}{R_{1,2}}\left[\left(x_{2}-x_{1}\right) \hat{x}+\left(y_{2}-y_{1}\right) \hat{y}+\left(z_{2}-z_{1}\right) \hat{z}\right]
$$

The oblique angle between two vectors was calculated as:

$$
\begin{gathered}
\alpha=\cos ^{-1}\left[\sin \left(\theta_{1}\right) \sin \left(\theta_{2}\right) \cos \left(\varphi_{1}\right) \cos \left(\varphi_{2}\right)+\sin \left(\theta_{1}\right) \sin \left(\theta_{2}\right) \sin \left(\varphi_{1}\right) \sin \left(\varphi_{2}\right)\right. \\
\left.+\cos \left(\theta_{1}\right) \cos \left(\theta_{2}\right)\right]
\end{gathered}
$$

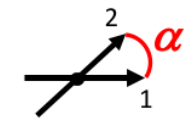

Extracted $J_{m, n}$ values and calculated geometric parameters for dye aggregates are summarized in the Tbls. S11 and S12. 
Table S6. Input fitting parameters used in calculations of squaraine and Cy5 aggregates.

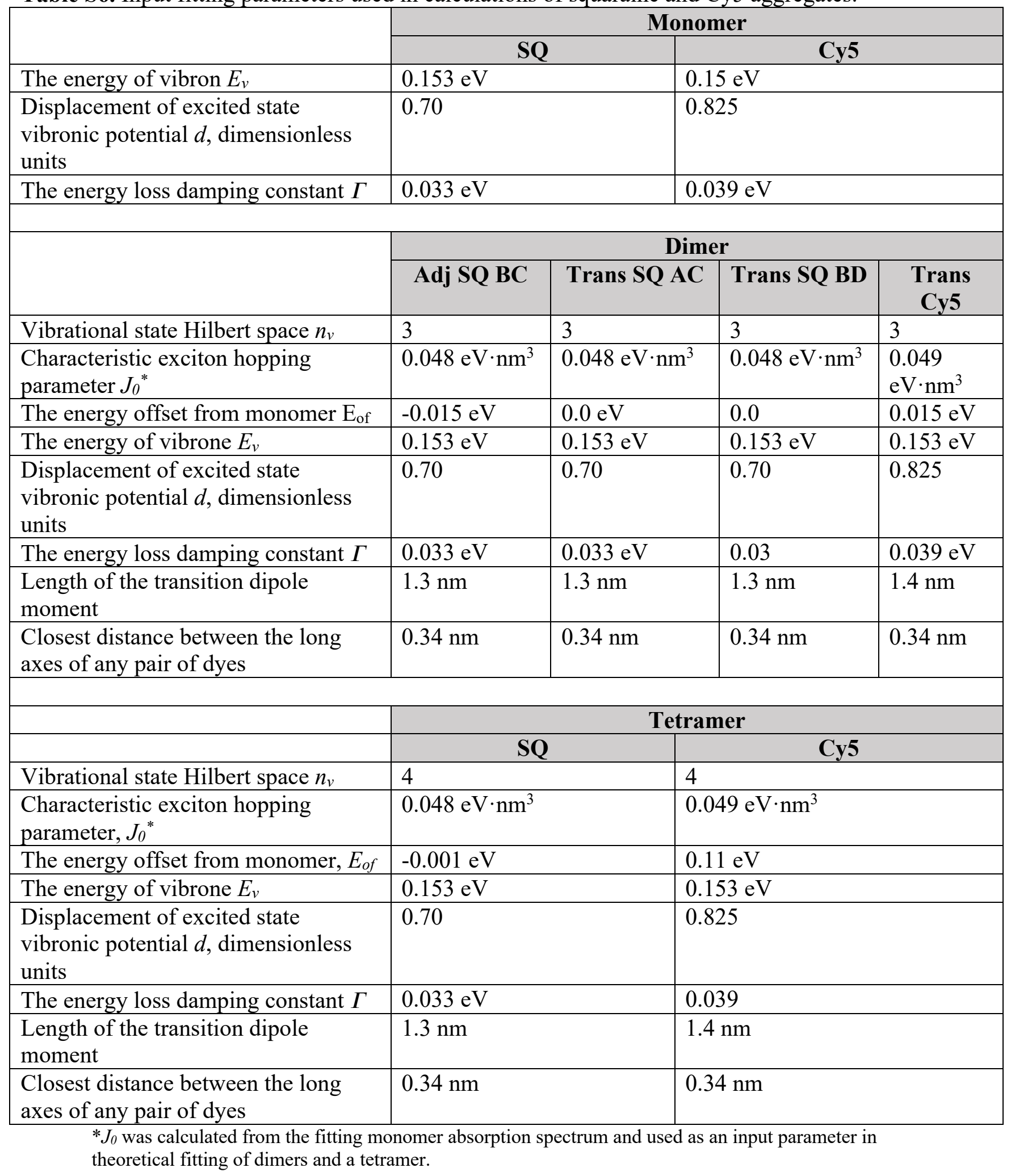


Table S7. Output parameters extracted from calculations of the monomer templated in DNA HJ.

\begin{tabular}{|l|l|l|}
\hline & \multicolumn{1}{|c|}{ SQ monomer } & \multicolumn{1}{c|}{ Cy5 monomer } \\
\hline Dipole moment $M_{01}$ & $11.68 \mathrm{Debye}$ & $11.85 \mathrm{Debye}$ \\
\hline Characteristic exciton hopping parameter $J_{0}$ & $0.048 \mathrm{eV} \cdot \mathrm{nm}^{3}$ & $0.049 \mathrm{eV} \cdot \mathrm{nm}^{3}$ \\
\hline
\end{tabular}

Table S8. Goodness of fit parameters for absorbance and CD spectra of squaraine aggregates.

\begin{tabular}{|c|c|c|c|c|c|c|c|}
\hline Construct & $r$ & $O I_{A B}$ & $O I_{C D}$ & $O I_{T o t}$ & $M S D_{a b s}$ & $M S D_{c d}$ & $w_{\text {abscd }}$ rms \\
\hline $\begin{array}{l}\text { SQ Adjacent } \\
\text { Dimer BC }\end{array}$ & 1.002 & 0.983 & 0.918 & 0.950 & 0.479 & 1.107 & 1.586 \\
\hline $\begin{array}{c}\text { SQ Transverse } \\
\text { Dimer AC }\end{array}$ & 1.049 & 0.969 & 0.968 & 0.968 & 0.888 & 0.428 & 1.316 \\
\hline $\begin{array}{c}\text { SQ Transverse } \\
\text { Dimer BD }\end{array}$ & 1.011 & 0.889 & 0.987 & 0.746 & 0.355 & 2.918 & 3.273 \\
\hline SQ Tetramer & 0.906 & 0.958 & 0.977 & 0.968 & 1.146 & 0.424 & 1.569 \\
\hline $\begin{array}{c}\text { Cy5 Transverse } \\
\text { Dimer }\end{array}$ & 1.009 & 0.984 & 0.950 & 0.967 & 0.339 & 1.242 & 1.581 \\
\hline Cy5 Tetramer & 1.036 & 0.968 & 0.587 & 0.778 & 0.407 & 6.843 & 7.250 \\
\hline
\end{tabular}

$\boldsymbol{r}$ - $\quad$ the ratio of theoretical to experimental values of the ratio of the max abs CD peak height to max absorbance peak height

$\boldsymbol{O I}_{\boldsymbol{A B}}$ - $\quad$ normalized overlap integral for the experimental and theoretical absorbance curves

$\boldsymbol{O I}_{\boldsymbol{C D}}$ - $\quad$ normalized overlap integral for the experimental and theoretical CD spectra

$\boldsymbol{O I}_{\text {tot }^{-}} \quad$ mean of $\boldsymbol{O I}_{A B}$ and $\boldsymbol{O I}_{C D}$

$\boldsymbol{M S D}_{\boldsymbol{a b s}}$ - absorbance spectrum mean-square deviation

$\boldsymbol{M S D}_{\boldsymbol{c} \boldsymbol{}}-\quad$ CD spectrum mean-square deviation

$\boldsymbol{w}_{\text {abscdrms }}$ - weighted mean-squared deviation between the experimental and theoretical abs and cd spectra 

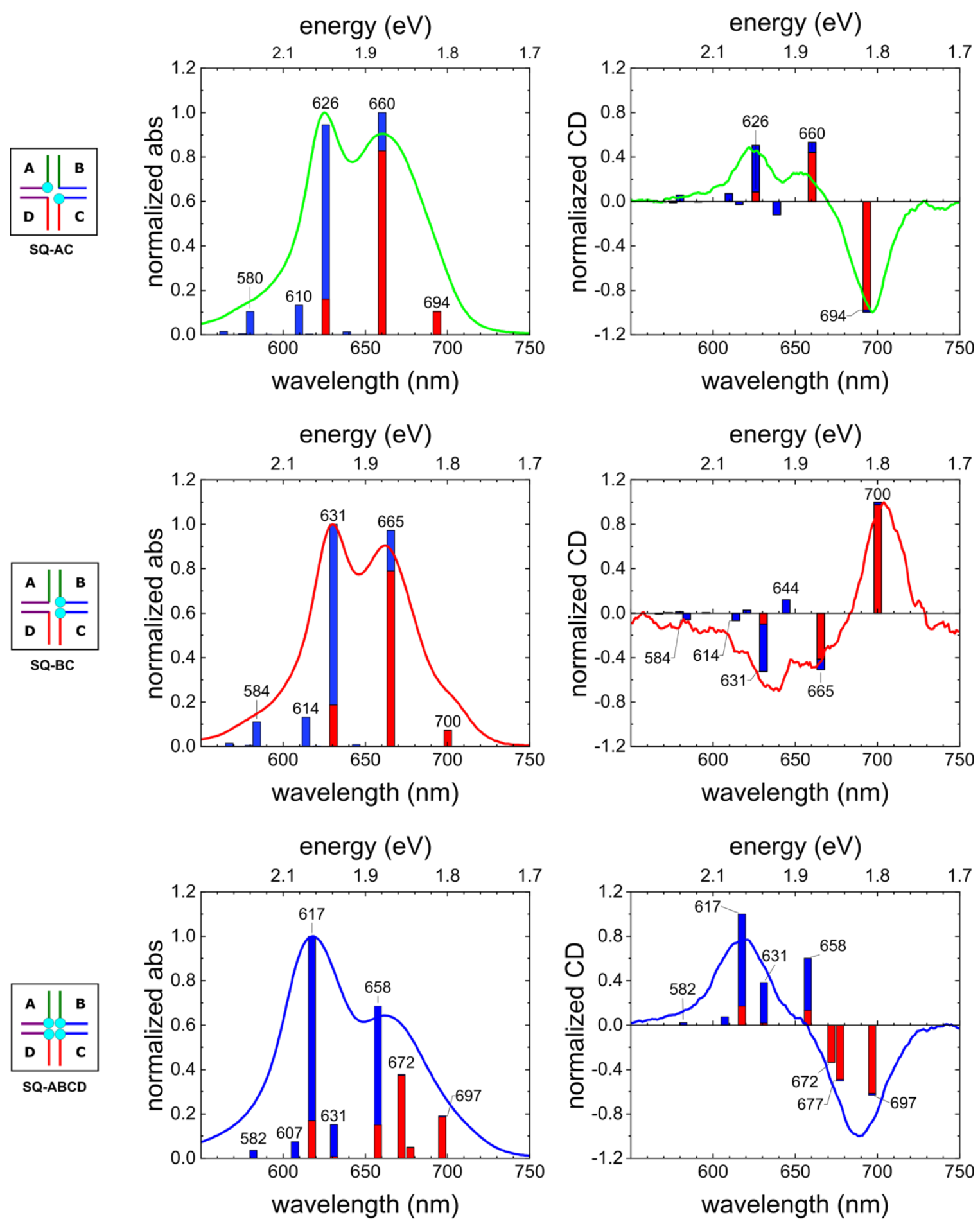

$\square$ electronic component

vibronic component

Figure S9. Electronic versus vibronic contribution to energy states in absorbance and CD spectra of the Square 660 adjacent dimer, transverse dimer and a tetramer obtained from the theoretical modeling. 


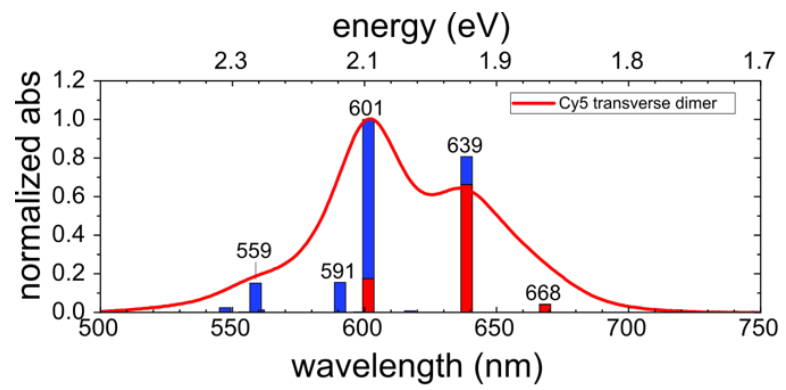

$\square$ electronic component

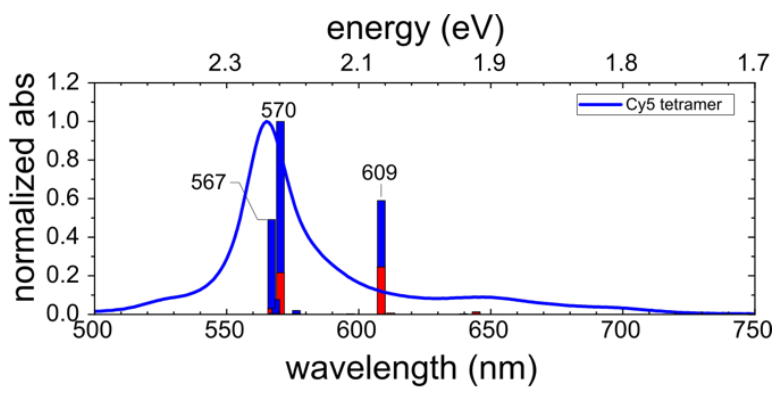

$\square$ vibronic component

Figure S10. Electronic versus vibronic contribution to energy states in absorbance of Cy5 transverse dimer and a tetramer obtained from the theoretical modeling.

Table S9. Kühn-Renger-May model fitting outputs describing each dye orientation and position in squaraine aggregates.

\begin{tabular}{|l|c|c|c|c|c|}
\hline Construct/Parameter & $\theta_{i}\left(^{\circ}\right)$ & $\varphi_{i}\left(^{\circ}\right)$ & $x_{i}(\AA)$ & $y_{i}(\AA)$ & $z_{i}(\AA)$ \\
\hline \multicolumn{7}{|c|}{ SQ Adjacent dimer BC } \\
\hline Dye 1 & 102.02 & 5.93 & 23.4 & -2.3 & 8.8 \\
\hline Dye 2 & 80.28 & 10.88 & 24.4 & -4.3 & 2.9 \\
\hline \multicolumn{7}{|c|}{ SQ Transverse dimer AC } \\
\hline Dye 1 & 11.74 & -2.10 & 0.71 & -1.02 & 3.3 \\
\hline Dye 2 & -14.94 & 0.10 & 0.10 & -8.2 & 2.3 \\
\hline \multicolumn{7}{|c|}{ SQ Transverse dimer BD } \\
\hline Dye 1 & 11.78 & -0.94 & -0.72 & 1.64 & -1.14 \\
\hline Dye 2 & -11.69 & 1.17 & -0.09 & 1.70 & -1.05 \\
\hline \multicolumn{7}{|c|}{ SQ Tetramer } \\
\hline Dye 1 & 95.99 & 26.92 & 1.47 & -9.1 & 12.9 \\
\hline Dye 2 & 83.05 & -18.27 & 1.53 & -12.9 & 17.6 \\
\hline Dye 3 & 80.57 & 7.74 & 1.37 & -17.7 & 21.6 \\
\hline Dye 4 & 82.17 & -5.11 & 2.13 & 2.9 & 17.7 \\
\hline
\end{tabular}

Table S10. Kühn-Renger-May model fitting outputs describing each dye orientation and position in $\mathbf{C y 5}$ aggregates.

\begin{tabular}{|l|c|c|c|c|c|}
\hline Construct/Parameter & $\theta_{i}\left({ }^{\circ}\right)$ & $\varphi_{i}\left({ }^{\circ}\right)$ & $x_{i}(\AA)$ & $y_{i}(\AA)$ & $z_{i}(\AA)$ \\
\hline \multicolumn{7}{|c|}{ Cy5 Transverse dimer } \\
\hline Dye 1 & 8.71 & -1.47 & -2.86 & -12.46 & 10.19 \\
\hline Dye 2 & -9.08 & -1.03 & -7.46 & -9.83 & 9.57 \\
\hline \multicolumn{7}{|c|}{ Cy5 Tetramer } \\
\hline Dye 1 & 83.06 & -0.16 & 9.53 & 12.25 & 10.47 \\
\hline Dye 2 & 94.22 & -1.21 & 9.97 & 15.54 & 9.39 \\
\hline Dye 3 & 82.93 & 0.04 & 15.53 & 15.28 & 21.34 \\
\hline Dye 4 & 89.36 & 0.68 & 13.82 & 11.47 & 21.71 \\
\hline
\end{tabular}


a

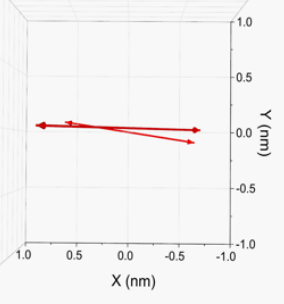

d

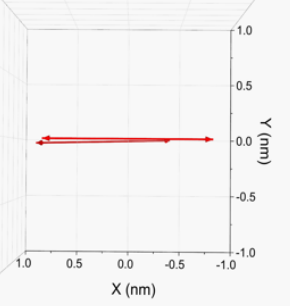

g

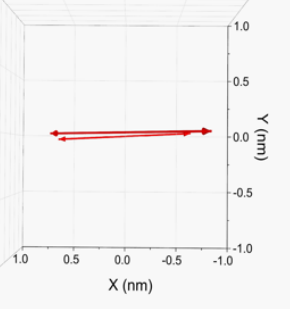

j

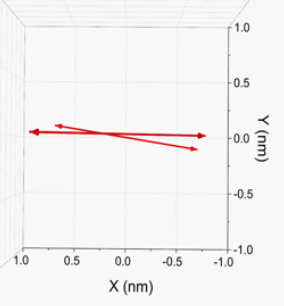

b

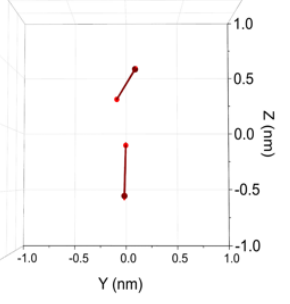

SQ-BD Transverse Dimer

e

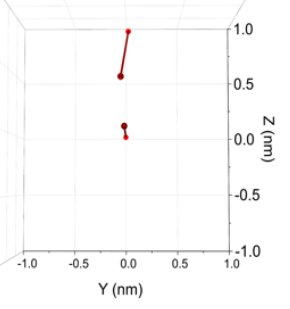

SQ-BC Adjacent Dimer

h

Cy5 Transverse Dimer $\mathrm{k}$
C

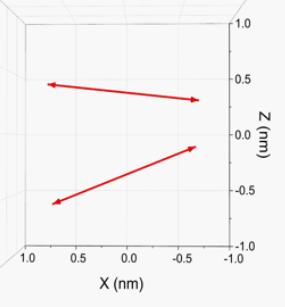

\section{$f$}

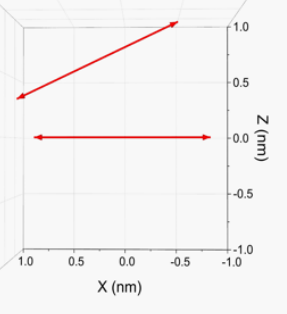

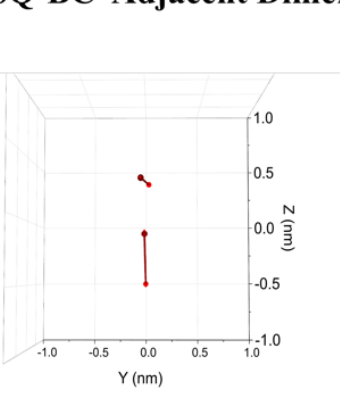

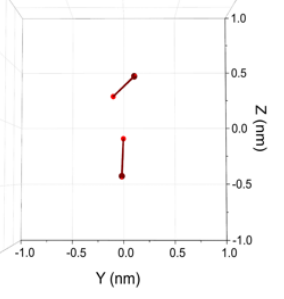

(1)
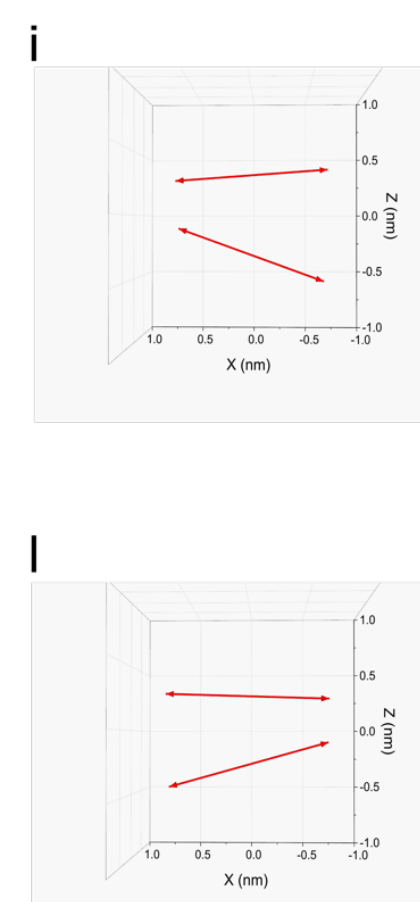

Figure S11. Transition dipole moments derived from the KRM modeling of the absorption and CD spectra of the Square-660 and Cy5 dimers. The transition dipole moments corresponding to each dye in a dimer are shown as red double-headed arrows projected into the XY, YZ, and XZ planes. 


\section{Cy5 Tetramer}

a
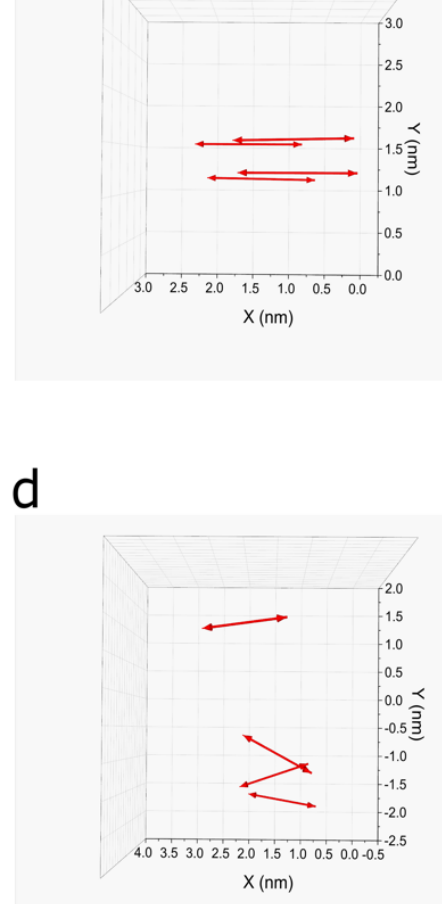

b

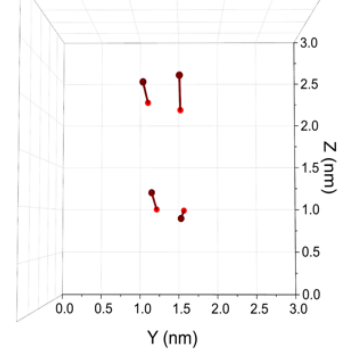

SQ Tetramer

e

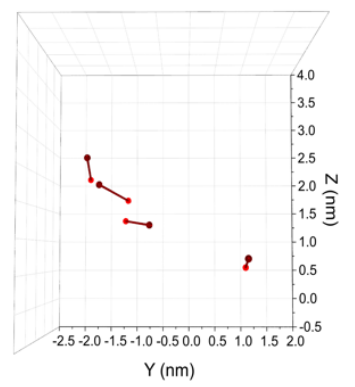

C

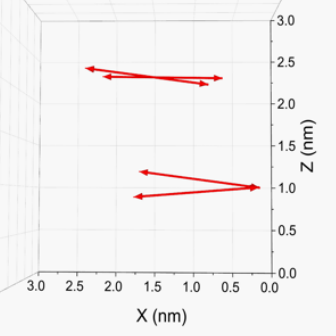

$f$

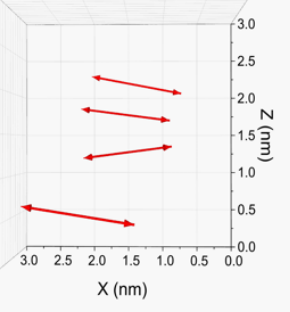

Figure S12. Transition dipole moments derived from the KRM modeling of the absorption and CD spectra of the Square-660 and Cy5 tetramers. The transition dipole moments corresponding to each dye in a tetramer are shown as red double-headed arrows projected into the XY, YZ, and $\mathrm{XZ}$ planes. 
Table S11. Calculated $J_{1,2}$ and geometric parameters for SQ Dimer Aggregates.

\begin{tabular}{|c|c|c|c|c|}
\hline Dimer Aggregate & $\begin{array}{l}\boldsymbol{J}_{\mathbf{1 , 2}} \\
(\mathbf{m e V})\end{array}$ & $\begin{array}{l}\text { Center-to Center } \\
\text { distance } \boldsymbol{R}(\mathbf{\AA})\end{array}$ & $\begin{array}{l}\text { Oblique angle } \\
\boldsymbol{\alpha}\left(\boldsymbol{(}^{\circ}\right)\end{array}$ & $\begin{array}{l}\text { Slip angle } \boldsymbol{\theta}_{\boldsymbol{s}} \\
\left({ }^{\circ}\right)\end{array}$ \\
\hline $\begin{array}{c}\text { SQ transverse } \\
\text { dimer AC }\end{array}$ & 65.4 & 6.48 & 26.7 & 70 \\
\hline $\begin{array}{c}\text { SQ transverse } \\
\text { dimer BD }\end{array}$ & 66.3 & 6.37 & 22.3 & 72 \\
\hline $\begin{array}{c}\text { SQ adjacent dimer } \\
\text { BC }\end{array}$ & 68 & 6.2 & 17.7 & 76 \\
\hline $\begin{array}{c}\text { Cy5 transverse } \\
\text { dimer }\end{array}$ & 70 & 5.3 & & \\
\hline
\end{tabular}

Table S12. Calculated $J_{m, n}$ and geometric parameters for SQ Tetramer aggregate.

Excitonic Hopping Parameter $J_{m, n}(\mathrm{meV})$

\begin{tabular}{|c|c|c|c|c|c|}
\hline$J_{1,2}$ & $J_{1,3}$ & $J_{1,4}$ & $J_{2,3}$ & $J_{2,4}$ & $J_{3,4}$ \\
\hline 66.2 & 15.4 & 3.5 & 50.8 & 6.8 & 2.8 \\
\hline \multicolumn{6}{|c|}{ Center-to-Center Distance $\boldsymbol{R}(\boldsymbol{\AA})$} \\
\hline$R_{1,2}$ & $R_{1,3}$ & $R_{1,4}$ & $R_{2,3}$ & $R_{2,4}$ & $R_{3,4}$ \\
\hline 6.1 & 12.3 & 14.6 & 6.4 & 17.0 & 22.3 \\
\hline \multicolumn{7}{|c|}{ Oblique angle $\left.\boldsymbol{\alpha} \mathbf{(}^{\circ}\right)$} \\
\hline$\alpha_{1,2}$ & $\alpha_{1,3}$ & $\alpha_{1,4}$ & $\alpha_{2,3}$ & $\alpha_{2,4}$ & $\alpha_{3,4}$ \\
\hline 46.7 & 24.6 & 34.8 & 25.9 & 13.1 & 12.8 \\
\hline \multicolumn{7}{|c|}{ Slip angle $\left.\boldsymbol{\theta}_{\boldsymbol{s}} \mathbf{(}^{\circ}\right)$} \\
\hline$\theta_{1,2}$ & $\theta_{1,3}$ & $\theta_{1,4}$ & $\theta_{2,3}$ & $\theta_{2,4}$ & $\theta_{3,4}$ \\
\hline 74 & 87 & 42 & 85 & 88 & 65 \\
\hline
\end{tabular}

Table S13. Calculated $J_{m, n}$ for Cy5 Tetramer aggregate.

\begin{tabular}{|c|c|c|c|c|c|}
\hline \multicolumn{7}{|c|}{ Excitonic Hopping Parameter $\boldsymbol{J}_{\boldsymbol{m}, \boldsymbol{n}}(\mathbf{m e V})$} \\
\hline$J_{1,2}$ & $J_{1,3}$ & $J_{1,4}$ & $J_{2,3}$ & $J_{2,4}$ & $J_{3,4}$ \\
\hline 98.6 & 8.9 & 13.2 & 9.9 & 10.7 & 83.2 \\
\hline \multicolumn{7}{|c|}{ Center-to-Center Distance $\boldsymbol{R}(\boldsymbol{A})$} \\
\hline$R_{1,2}$ & $R_{1,3}$ & $R_{1,4}$ & $R_{2,3}$ & $R_{2,4}$ & $R_{3,4}$ \\
\hline 3.6 & 12.6 & 11.8 & 13.2 & 13.5 & 4.2 \\
\hline
\end{tabular}


To build molecular models of dye aggregates, chemical structures of the core squaraine and Cy5 were created in ChemDraw 18.2, the coordinates to each dye were assigned in Avogadro 1.2.0. Chimera 1.14 was used to show mutual orientation of dyes in the aggregates.
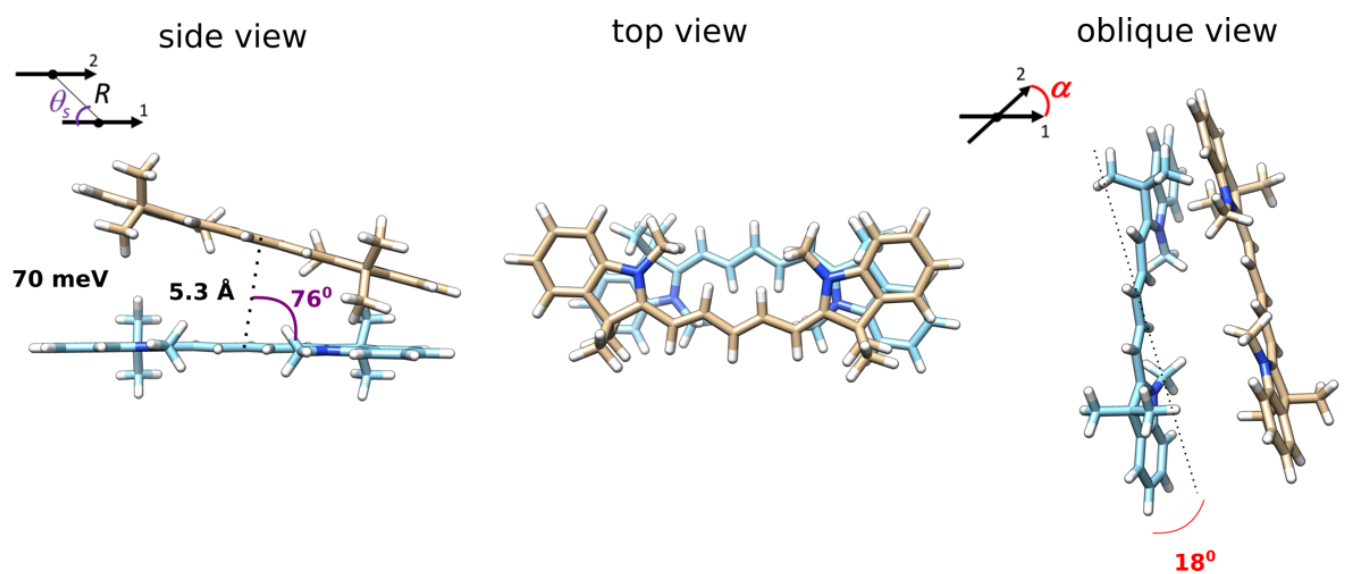

Figure S13. Molecular models of the Cy5 $\mathrm{H}$-dimer (transverse dimer). The side view shows a center-to-center distance $R$ in $\AA$ and a slip angle $\theta_{s}{ }^{\circ}$. The top view shows oblique angle $\alpha^{\circ}$ as an angle between vectors 1 and 2 if their centers are superimposed. Note, that the fitting procedure determines the position and orientation of the long axes of the Cy5 dyes, but not the rotation of the dye core around its long axis. As such, the dye core rotations were arbitrary chosen.

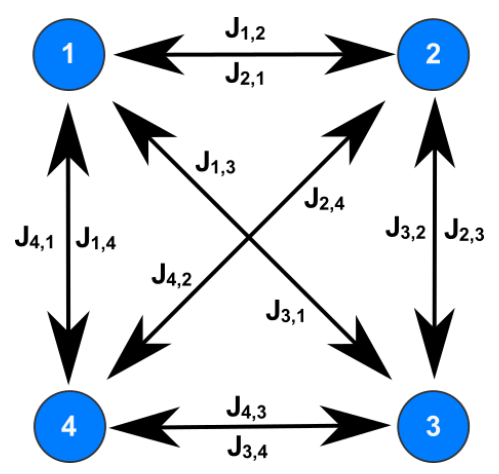

Figure S14. A matrix for excitonic hopping parameter $J_{m, n}$ in a tetramer. 


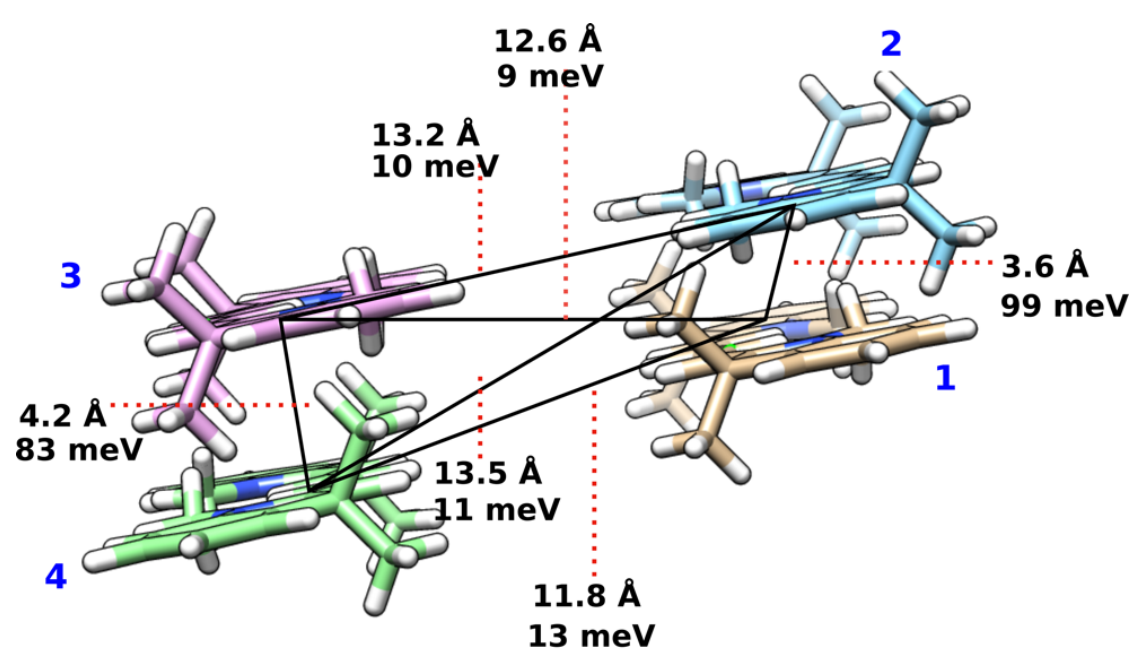

Figure S15. A molecular model of the Cy5 tetramer showing a center-to-center distance $R$ in $\AA$ and $J_{m, n}$ between each pait of dyes in meV. Note, that the fitting procedure determines the position and orientation of the long axes of the Cy5 dyes, but not the rotation of the dye core around its long axis. As such, the dye core rotations were arbitrary chosen.

Supporting Information 10. Influence of DNA HJ Conformations on Squaraine aggregation

In the presence of divalent cations such as $\mathrm{Mg}^{2+}$, unmodified DNA Holliday Junction (DNA HJ) maintains a stacked conformation. ${ }^{10}$ In the stacked conformation, two arms of HJ are stacked onto other two arms forming two coaxial continuous duplexes. The conformation is known to undergo a conformational interconversion which can be visualized by a two-state model where a transition between two stacking isomer iso I and iso II occurs through the open square conformation (Fig. S16a). ${ }^{10-11}$ In one stacking isomer (e.g. iso I), strands B and D adopt continuous helical structures while other two strands $\mathrm{A}$ and $\mathrm{C}$ are the crossover strands. In the second stacking isomer (then iso II), the strand folds are opposite. The stacked structure is stabilized by coaxial base pair stacking in the crossover area (junction point) which can also include van der Waals forces, hydrogen bonding, and water bridges. ${ }^{12}$ Thus, one stacking isomer often dominates, and this dominance is sequence dependent. (In Fig. S16a, iso II is arbitrarily 
chosen to be the major isomer.) In contrast, in the presence of monovalent cations such as $\mathrm{Na}^{+}$ (with the exception of very high concentration of $\mathrm{Na}^{+} \sim 1 \mathrm{M}$ ), ${ }^{13}$ the $\mathrm{HJ}$ primarily adopts an open square conformation with the arms extended.

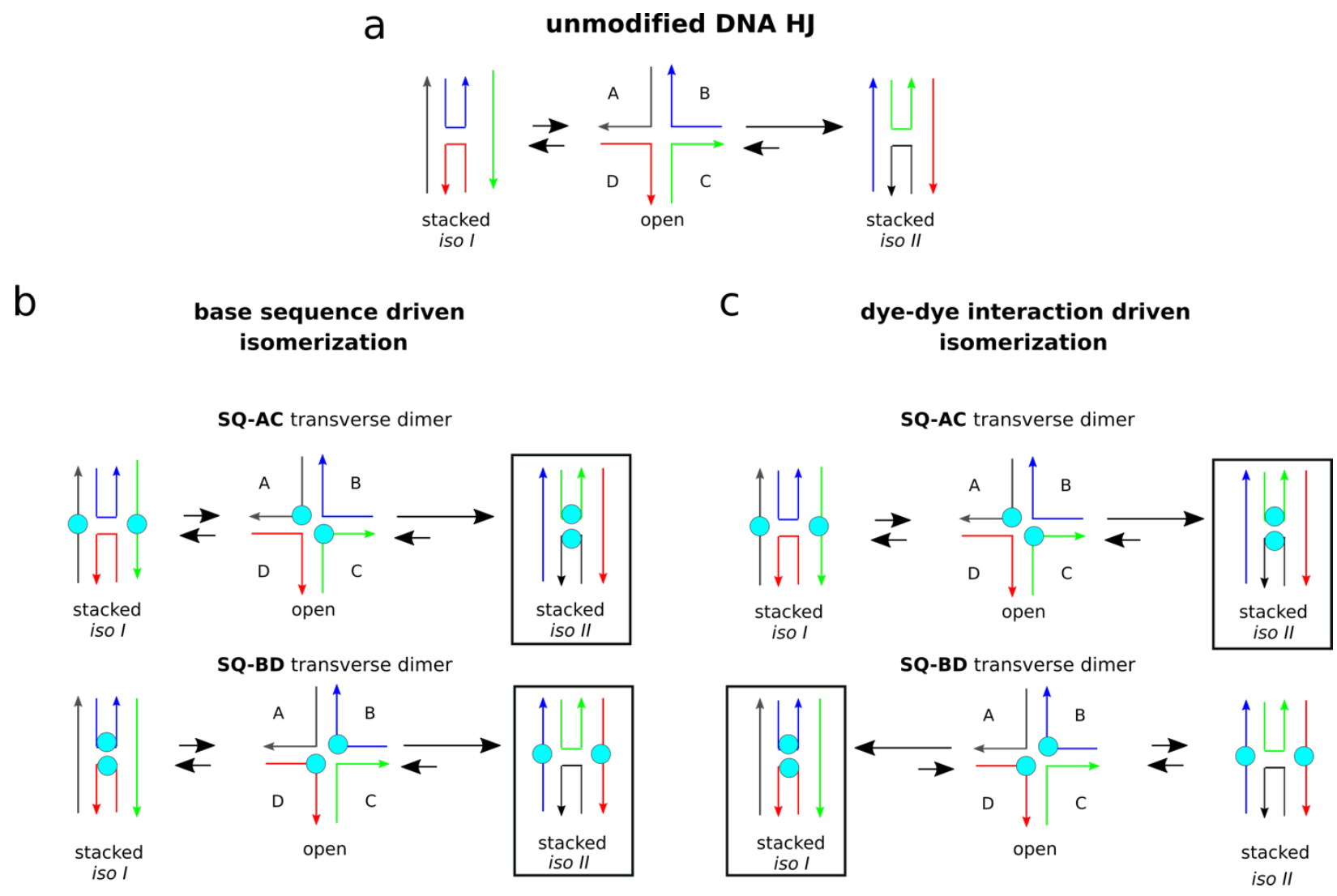

Fig. S16. (a) Unmodified DNA Holliday junction exists as a mixture of two stacked isomers: iso $I$ and iso II. The iso II conformer is arbitrarily chosen to be the major conformer. (b) Transverse dimers SQ-AC and SQ-BD assuming the same stacked isomer of DNA HJ dominates due to base sequence. (c) Transverse dimers SQ-AC and SQ-BD assuming the dominant stacked isomer is controlled by favorable dye-dye interactions.

The thermal denaturation experiments (Section S3) indicate that DNA HJ templating squaraine constructs in $1 \times \mathrm{TBE} 15 \mathrm{mM} \mathrm{MgCl}_{2}$ largely adopt a stacked conformation. Hence, the conformational isomerization between iso I and iso II isomers of the stacked DNA HJ might lead to a structural heterogeneity in the dye aggregates templated via DNA HJ. To estimate the 
influence of the stacked DNA HJ isomerization on the orientation of squaraines in their DNA HJ-templated aggregates, we constructed and examined an additional aggregate SQ-BD transverse dimer. In the SQ-BD transverse dimer, the dyes are positioned in a transverse fashion on the opposing strands B and D whereas in the initially constructed transverse dimer SQ-AC the dyes are positioned on the strands $\mathrm{A}$ and $\mathrm{C}$. We assumed that if interconversion between two isomers of the stacked conformation took place in the dye-modified DNA HJ the same way as it is known to take place in the unmodified DNA HJ, i.e. the same dominant isomer templated both dimers (e. g. iso II), then the geometries SQ-AC and SQ-BD dimers would be distinct (Fig. S16b). In particular, in the SQ-AC dimer, the dyes would be expected to be on the very proximate crossover strands in the dominant iso II isomer. (For reference, in unmodified DNA HJ, the HJ cavity is very compact with the crossover strands about $3.5 \AA$ apart. $)^{12}$ And in the SQBD dimer, the dyes would be expected to be on the continuous strands which are more distant in the same dominant iso II isomer. For this reason, the absorption of the SQ-BD dimer would be expected to be similar to the absorption of the transverse dimer templated via the open DNA HJ in the presence of $\mathrm{NaCl}$ (Fig. S17). In contrast, if the dominant isomer of the stacked DNA HJ conformation was governed by the dye interactions, the geometries of two dimers SQ-AC and SQ-BC would be anticipated to be similar (Fig. S16c). 

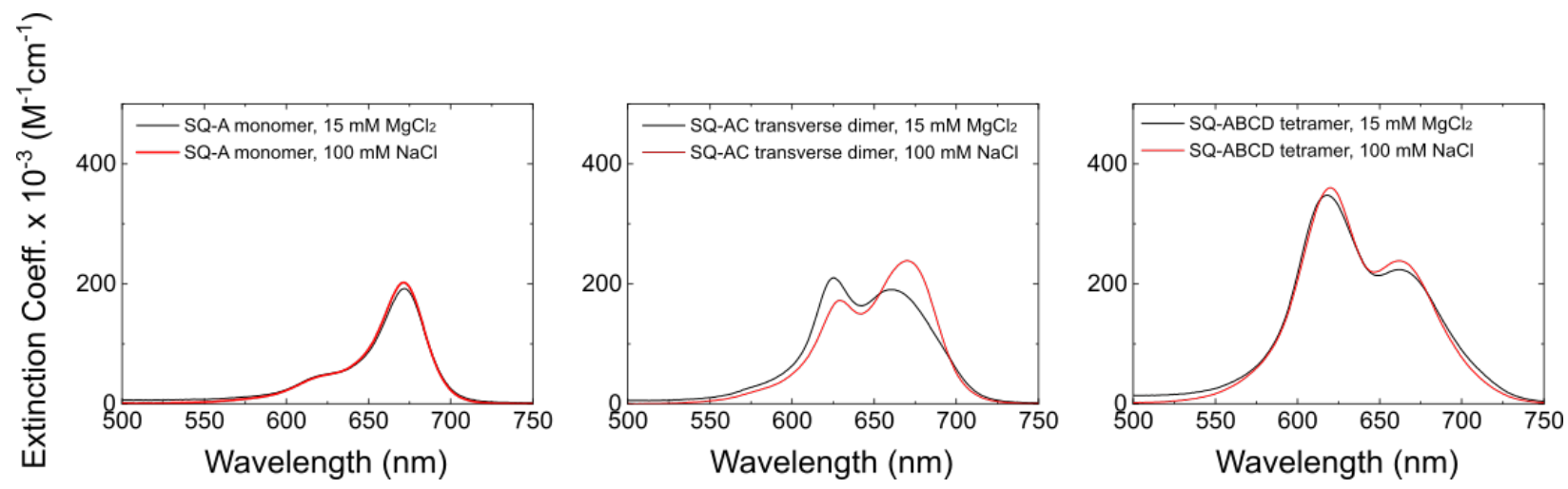

Fig. S17. Acquired steady-state absorption spectra of the DNA-Square 660 dye constructs in $1 \times$ TBE $100 \mathrm{mM} \mathrm{NaCl}$ (red line) and in $1 \times \mathrm{TBE} 15 \mathrm{mM} \mathrm{MgCl}_{2}$ (black like) at room temperature The DNA-dye construct concentration was $1.5 \mu \mathrm{M}$.

As the absorbance and CD data show, the synthesized SQ-BD dimer exhibited spectral profiles very similar to that of the SQ-AC dimer (Fig. S18). The KRM modeling of the SQ-BD dimer resulted in nearly identical center-to-center distance, geometry and coupling strength compared to the SQ-AC dimer (see Section S9). These results suggest that, potentially driven by dye-dye interactions, the squaraine dimer aggregation yields a conformational bias toward one isomer of the stacked DNA HJ conformation. Since absorption spectra of the transverse dimers SQ-AC and SQ-BD in $\mathrm{MgCl}_{2}$ are noticeably different from that of the transverse dimer in $\mathrm{NaCl}$, it is likely that the dominant stacked isomer is the one with the dyes positioned close on the crossover strands (i.e. iso II conformer for AC dimer and iso I conformer for BD dimer). The hydrophobic interaction between short hydrophobic spacers might also contribute toward the preference for the dyes to be on the proximate crossover strands. 

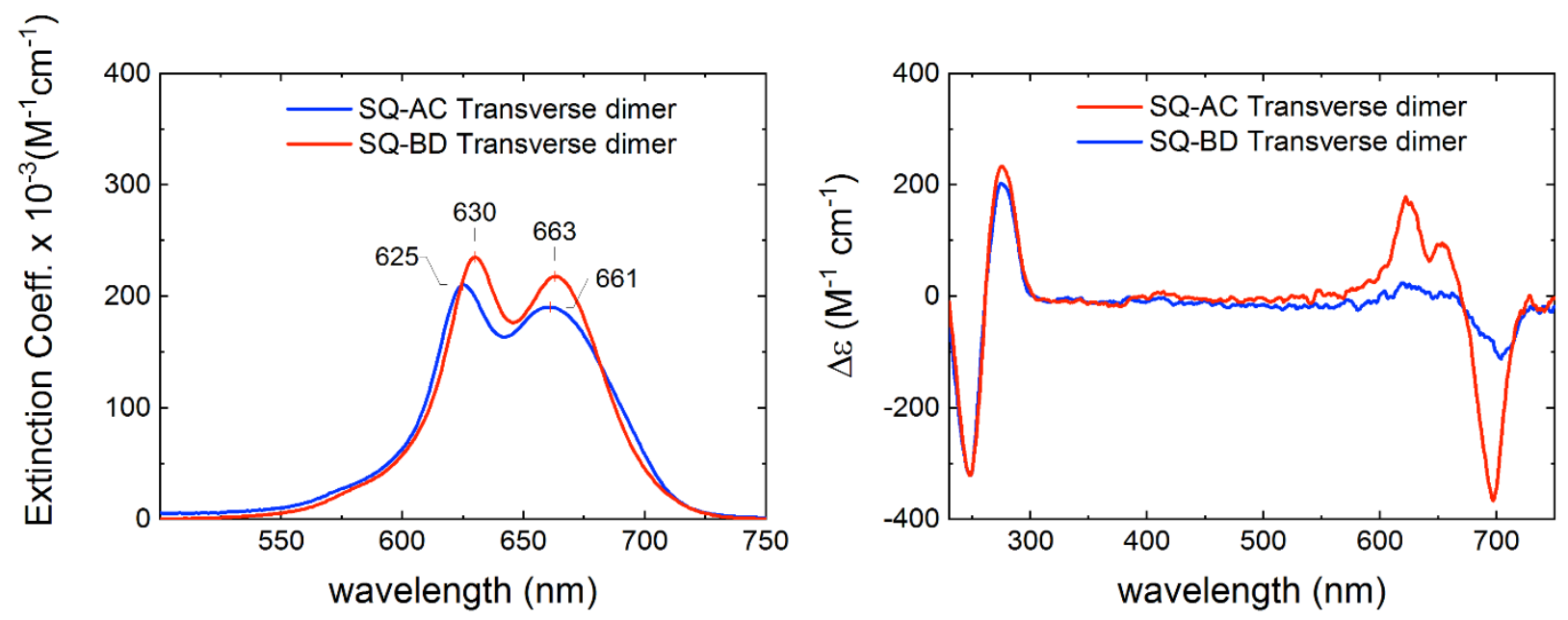

Fig. S18. (left panel) Acquired steady-state absorption spectra of the DNA-Square 660 transverse dimers $\mathrm{AC}$ (blue line) and $\mathrm{BD}$ (red line) in $1 \times \mathrm{TBE} 15 \mathrm{mM} \mathrm{MgCl} 2$ at room temperature. The DNA-dye construct concentration was $1.5 \mu \mathrm{M}$. (right panel) Acquired CD spectra of the DNASquare 660 transverse dimers $\mathrm{AC}$ (red line) and $\mathrm{BD}$ (blue line) in $1 \times \mathrm{TBE} 15 \mathrm{mM} \mathrm{MgCl} 2$ at room temperature. The DNA-dye construct concentration was $1.5 \mu \mathrm{M}$.

Due to the presence of dyes on each strand of the tetramer, the tetramer aggregation is not sensitive to the dominant isomer of the stacked DNA HJ. Moreover, the tetramer aggregation appears not to be sensitive to conformation of DNA HJ. For example, the spectral properties of the SQ-ABCD tetramer in the presence of $\mathrm{MgCl}_{2}$ and $\mathrm{NaCl}$ (Fig. S17 right) are nearly identical. This result suggests that dye-dye interactions are strong enough to drive tetramer aggregation regardless of DNA HJ conformation (stacked vs open). According to the melting point profiles, the tetramer in $\mathrm{NaCl}$ is templated by the open $\mathrm{DNA} \mathrm{HJ}$, while the same tetramer in the $\mathrm{MgCl}_{2}$ is templated by the stacked DNA HJ. Despite the difference in the conformation of the templating DNA HJ, the absorption profiles of both tetramers are strikingly similar. Also similar are the second melting transitions of the tetramer in $\mathrm{NaCl}\left(\mathrm{T}_{\mathrm{m}}=62.7{ }^{\circ} \mathrm{C}\right)$ and in the $\mathrm{MgCl}_{2}\left(\mathrm{~T}_{\mathrm{m}}=\right.$ $64.2^{\circ} \mathrm{C}$ ). Both of these similarities indicate that aggregation in the squaraine tetramers is strongly driven by dye-dye interactions and largely independent from the DNA HJ conformation. One possibility is that stacked HJ locally expands upon a linker insertion in each strand, thus 
adopting more parallelogram-like geometry to accommodate the squaraine tetramer. On the other hand, the open conformation of DNA HJ might adopt a more compact geometry to promote dye interactions in the tetramer.

\section{Supporting Information 11: References}

1. Shida, T.; Iwasaki, H.; Shinagawa, H.; Kyogoku, Y. Characterization and Comparison of Synthetic Immobile and Mobile Holliday Junctions1. J. Biochem. 1996, 119, 653-658.

2. $\quad$ Carr, C. E.; Marky, L. A. Melting Behavior of a DNA Four-Way Junction Using Spectroscopic and Calorimetric Techniques. J. Am. Chem. Soc. 2017, 139, 14443-14455.

3. Huff, J. S.; Davis, P. H.; Christy, A.; Kellis, D. L.; Kandadai, N.; Toa, Z. S. D.; Scholes, G. D.; Yurke, B.; Knowlton, W. B.; Pensack, R. D. DNA-Templated Aggregates of Strongly Coupled Cyanine Dyes: Nonradiative Decay Governs Exciton Lifetimes. J. Phys. Chem. Lett. 2019, 10, 2386-2392.

4. Abramavicius, D.; Palmieri, B.; Mukamel, S. Extracting Single and Two-Exciton Couplings in Photosynthetic Complexes by Coherent Two-Dimensional Electronic Spectra. Chem. Phys. 2008, 357, 79-84.

5. Abramavicius, D.; Mukamel, S. Exciton Dynamics in Chromophore Aggregates with Correlated Environment Fluctuations. J. Chem. Phys. 2011, 134, 174504.

6. Abramavicius, D.; Palmieri, B.; Voronine, D. V.; Šanda, F.; Mukamel, S. Coherent Multidimensional Optical Spectroscopy of Excitons in Molecular Aggregates; Quasiparticle Versus Supermolecule Perspectives. Chem. Rev. 2009, 109, 2350-2408.

7. Czikklely, V.; Forsterling, H. D.; Kühn, H. Extended Dipole Model for Aggregates of Dye Molecules. Chem. Phys. Lett. 1970, 6, 207-210.

8. Holstein, T. Studies of Polaron Motion: Part I. The Molecular-Crystal Model. Ann. Phys. 1959, $8,325-342$.

9. $\quad$ Chung, P.-H.; Tregidgo, C.; Suhling, K. Determining a Fluorophore's Transition Dipole Moment from Fluorescence Lifetime Measurements in Solvents of Varying Refractive Index. Methods Appl. Fluoresc. 2016, 4, 045001.

10. Lilley, D. M. J.; Norman, D. G. The Holliday Junction Is Finally Seen with Crystal Clarity. Nat. Struct. Biol. 1999, 6, 897-899.

11. Hyeon, C.; Lee, J.; Yoon, J.; Hohng, S.; Thirumalai, D. Hidden Complexity in the Isomerization Dynamics of Holliday Junctions. Nat. Chem. 2012, 4, 907-914.

12. Ortiz-Lombardía, M.; González, A.; Eritja, R.; Aymamí, J.; Azorín, F.; Coll, M. Crystal Structure of a DNA Holliday Junction. Nat. Struct. Biol. 1999, 6, 913-917.

13. Joo, C.; McKinney, S. A.; Lilley, D. M.; Ha, T. Exploring Rare Conformational Species and Ionic Effects in DNA Holliday Junctions Using Single-Molecule Spectroscopy. J. Mol. Biol. 2004, 341, 739-751. 